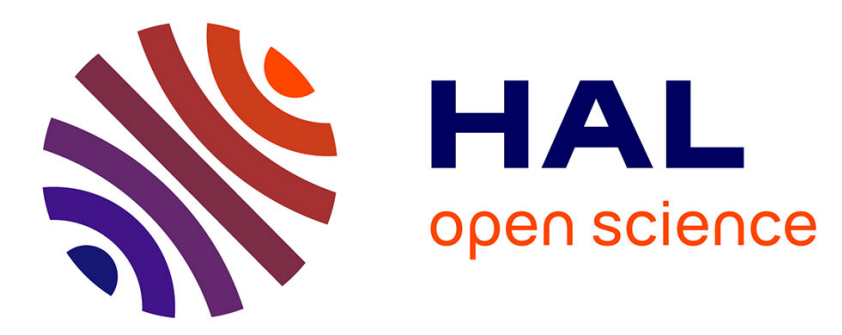

\title{
Low-Complexity Algorithms for Low Rank Clutter Parameters Estimation in Radar Systems
}

Ying Sun, Arnaud Breloy, Babu Prabhu, Daniel P. Palomar, Frédéric Pascal, Guillaume Ginolhac

\section{- To cite this version:}

Ying Sun, Arnaud Breloy, Babu Prabhu, Daniel P. Palomar, Frédéric Pascal, et al.. Low-Complexity Algorithms for Low Rank Clutter Parameters Estimation in Radar Systems. IEEE Transactions on Signal Processing, 2016, 64 (8), pp.1986-1998. 10.1109/TSP.2015.2512535 . hal-01377663

\section{HAL Id: hal-01377663 https://hal.science/hal-01377663}

Submitted on 26 Feb 2020

HAL is a multi-disciplinary open access archive for the deposit and dissemination of scientific research documents, whether they are published or not. The documents may come from teaching and research institutions in France or abroad, or from public or private research centers.
L'archive ouverte pluridisciplinaire HAL, est destinée au dépôt et à la diffusion de documents scientifiques de niveau recherche, publiés ou non, émanant des établissements d'enseignement et de recherche français ou étrangers, des laboratoires publics ou privés. 


\title{
Low-Complexity Algorithms for Low Rank Clutter Parameters Estimation in Radar Systems
}

\author{
Ying Sun, Arnaud Breloy, Student Member, IEEE, Prabhu Babu, Daniel Palomar, Fellow, IEEE, Frédéric \\ Pascal, Senior Member, IEEE, Guillaume Ginolhac, Member, IEEE
}

\begin{abstract}
This paper addresses the problem of the clutter subspace projector estimation in the context of a disturbance composed of a low rank heterogeneous (Compound Gaussian) clutter and white Gaussian noise. In such a context, adaptive processing based on an estimated orthogonal projector onto the clutter subspace (instead of an estimated covariance matrix) requires less samples than classical methods. The clutter subspace estimate is usually derived from the Singular Value Decomposition of a covariance matrix estimate. However, it has been previously shown that a direct Maximum Likelihood Estimator of the clutter subspace projector can be obtained for the considered context. In this paper, we derive two algorithms based on the block majorization-minimization framework to reach this estimator. These algorithms are shown to be computationally faster than the state of the art, with guaranteed convergence. Finally, the performance of the related estimators is illustrated on realistic Space Time Adaptive Processing for airborne radar simulations.
\end{abstract}

Index Terms-Covariance Matrix and Projector estimation, Maximum Likelihood Estimator, Low Rank structure, Compound Gaussian, majorization-minimization.

\section{INTRODUCTION}

$\mathbf{I}$ $\mathrm{N}$ array processing, many applications require the use of the covariance matrix $(\mathrm{CM})$ of the noise; these include source localization techniques [1], [2], radar and sonar detection methods [3], [4], and filters [5]. In practice, the CM is unknown and has to be estimated from a set of samples $\mathbf{z}_{k} \in \mathbb{C}^{M}, k \in \llbracket 1, K \rrbracket$, which are $K$ signal-free independent realizations of the noise. The $\mathrm{CM}$ estimate is then used to perform the so-called adaptive process. In radar systems, the noise is composed of a correlated noise, referred to as clutter (caused by the response of the environment to the emitted signal), and White Gaussian Noise (WGN, the thermal noise due to electronics). The total covariance of this disturbance is therefore

$$
\boldsymbol{\Sigma}_{t o t}=\boldsymbol{\Sigma}+\sigma^{2} \mathbf{I}
$$

where $\boldsymbol{\Sigma}$ is the clutter $\mathbf{C M}$ and $\sigma^{2} \mathbf{I}$ is the CM of the WGN. In most cases, the clutter belongs to a subspace of limited

Ying Sun, Prabhu Babu, and Daniel P. Palomar are with the Hong Kong University of Science and Technology (HKUST), Hong Kong. e-mail: ysunac, eeprabhubabu, palomar@ust.hk. Arnaud Breloy is with the SATIE, ENS Cachan, CNRS, F-94230 Cachan, and with SONDRA, Centrale-Supélec, F91192 Gif-sur-Yvette Cedex, France (e-mail: abreloy@satie.ens-cachan.fr). Guillaume Ginolhac is with the LISTIC, Universite de Savoie Mont-Blanc, 74944 Annecy le Vieux Cedex, France (e-mail: guillaume.ginolhac@univsavoie.fr). Frédéric Pascal is with L2S, Centrale-Supélec, F-91192 Gif-surYvette Cedex, France (e-mail: frederic.pascal@centralesupelec.fr).

The work of Ying Sun, Prabhu Babu, and Daniel P. Palomar was supported by the Hong Kong RGC 16207814 research grant. The work of Arnaud Breloy was supported by a DGA grant and the SONDRA PA10-DSOCO11127. dimension, meaning that the clutter $\mathrm{CM} \Sigma$ has rank $R<M$. Consequently, the total CM is structured as a Low Rank (LR) plus a scaled identity matrix.

When the clutter corresponds to a strong interference contained in a low dimensional subspace $(R \ll M)$, one can use the following LR approximation [6], [7]:

$$
\boldsymbol{\Sigma}_{t o t}^{-1} \simeq \frac{1}{\sigma^{2}} \boldsymbol{\Pi}_{c}^{\perp} \simeq \frac{1}{\sigma^{2}}\left(\mathbf{I}-\boldsymbol{\Pi}_{c}\right)
$$

where $\Pi_{c}$, named clutter subspace projector, is the orthogonal projector onto the clutter subspace. This subspace is spanned by the $R$ eigenvectors $\mathbf{v}_{r}$ associated with the $R$ largest eigenvalues of the matrix $\boldsymbol{\Sigma}$, i.e., $\boldsymbol{\Pi}_{c}=\sum_{r=1}^{R} \mathbf{v}_{r} \mathbf{v}_{r}^{H}$. This approximation allows developing an adaptive process (e.g., filter or detector) that relies on a clutter subspace projector estimate $\hat{\boldsymbol{\Pi}}_{c}$ rather than a total CM estimate $\hat{\boldsymbol{\Sigma}}_{t o t}$. Note that in this paper, we consider that the clutter rank $R$ is known or fixed from a prior estimation step. There are several methods of rank estimation present in the literature (e.g., [8]-[10] and references therein). Moreover, in some applications, the clutter rank can be directly derived from the geometry of the system (such as in STAP [11] thanks to the Brennan rule [12]).

The practical use of the LR approximation is that an adaptive LR process requires less samples to reach the equivalent performance to the classical ones, which is valuable since the number of available samples is often limited. For example, if we consider the case of a Gaussian distributed clutter, the optimal adaptive filter is built from the Sample Covariance Matrix (SCM), which is the Maximum Likelihood Estimator (MLE) in that scenario. In this case, $K=2 M$ samples are required to ensure a $3 \mathrm{~dB}$ loss of the output Signal to Noise Ratio (SNR) compared to the optimal non-adaptive filter [13]. If instead we use the MLE of the clutter subspace projector, which corresponds to the subspace spanned by the $R$ largest eigenvectors of the SCM and can be obtained from its EigenValue Decomposition (EVD), one can build an adaptive LR filter that reaches the equivalent performance to the previous scheme with only $K=2 R \ll 2 M$ samples [14].

However, it is now well-known that most modern radar clutter measurements are not Gaussian and behave heterogeneously. Therefore, the SCM may not provide the optimal solution since it is not an accurate estimator of the $\mathrm{CM}$ for heavy-tailed distributions or in the presence of outliers. To account for the heterogeneity of the clutter, one can model it with a Compound Gaussian distribution, which is a sub-familly of the Complex Elliptically Symmetric (CES) distribution [15]. The Compound Gaussian family covers a large panel of well- 
known and useful distributions, notably Gaussian, Weibull, K-distribution, $t$-distribution, etc. Moreover, it has a wellfounded physical interpretation and presents good agreement with several real data sets [16]-[19]. Eventually, the total disturbance will be modeled in this paper as an LR Compound Gaussian clutter plus a WGN (as done in [7], [20]-[22]).

We point out that the sum of a Compound Gaussian and a WGN cannot be represented as a simple Compound Gaussian vector with different distribution parameters (except for the trivial Gaussian case). Nevertheless, most of the related works consider the case of a full rank Compound Gaussian clutter and neglect the possible LR plus WGN structure. Under this framework, robust estimation ${ }^{1}$ of the $\mathrm{CM}$ can be performed using the so-called $M$-estimators [23]-[25] that are seen as generalized MLEs for CES distributions. A detailed review of this framework can be found in [15]. These estimators have been studied in detail and successfully applied in modern detection/estimation literature due to their interest both from a theoretical and an application point of view [26]-[32]. To cope with an under-sampled scenario, regularization of these estimators has been studied intensively in the literature, and is still an on-going cutting-edge topic. For example, shrinkage estimators for the CES model have been considered in [33] [36] that shrink the estimator to the identity matrix. Regularization by imposing constraints on the condition number of the covariance matrix for the compound Gaussian model has been studied in [37]. Another class of works tackle this problem by imposing structural constraints, including group symmetry, Toeplitz, Kronecker structures, on the covariance matrix, see [37]-[41] for example. Instead of the SCM, it is possible to derive clutter subspace projector estimators from the EVD of a (regularized or not) $M$-estimator. However, despite their robustness properties, $M$-estimators may not achieve the optimal clutter subspace estimation performance as they do not take into account the noise structure (1) that we consider in this work.

For this noise model, the seminal work [21] derived the MLE of the clutter subspace projector under the assumptions that the CM of the LR Compound Gaussian clutter has identical eigenvalues, and the Probability Density Function (PDF) of the texture is known. The assumption of known texture PDF has been relaxed in [42] by treating the texture as an unknown deterministic parameter. The expression of the MLE of the clutter CM parameters (eigenvectors and eigenvalues) was derived in [43], which does not have a simple closed-form and therefore requires an iterative algorithm to be reached. Initially, [43] focused on the clutter subspace projector MLE and proposed an ad-hoc algorithm to obtain it. This leads to an accurate clutter subspace projector estimator, but a poor recovery of the total CM. In [44], the high Clutter to Noise Ratio (CNR) assumption was made to derive a general "2Step MLE" algorithm (with several possible adaptations) that was shown to provide accurate estimates of the CM. Most algorithms developed [43], [44] require the use of a gradient descent algorithm on the manifold proposed in [45], which can be difficult to tune and computationally costly.

\footnotetext{
${ }^{1}$ i.e., not highly sensitive to the underlying distribution
}

In this paper, we propose to apply the block majorizationminimizaton algorithm framework to the problem of computing the considered MLE. We derive two new algorithms that enjoy the following properties:

- They do not rely on any heuristic (as in [43]) or high CNR assumption (as in [44]).

- They are computationally faster than the ones derived in [43] and [44], hence more suitable for implementation.

- Compared to the gradient descent algorithm [45], which requires many trials to find the descent step, the proposed algorithms only require basic matrix operations or Singular Value Decomposition (SVD).

The paper is organized as follows. Section II states the problem formulation and briefly reviews the block majorizationminimizaton methodology. Then, Sections III and IV derive the two new algorithms for computing the MLE of the considered problem. The difference between the two algorithms lies in the choice of the variables with respect to which the likelihood function is cyclically optimized. In section $\mathrm{V}$, we discuss possible methods for of estimating the clutter rank and the white Gaussian noise power, which are assumed to be known in this study. Section VI validates the performance of the proposed algorithms with simulations. Moreover, the proposed algorithms are applied to a realistic Space Time Adaptive Processing (STAP) for airborne radar simulation. Finally, Section VII draws the conclusions of this study.

Throughout the paper the following conventions are adopted: italic indicates a scalar quantity, lower case boldface indicates a vector quantity, and upper case boldface a matrix. $H$ denotes the transpose conjugate operator or the simple conjugate operator for a scalar quantity. ${ }^{T}$ denotes the transpose operator. $\mathrm{d}(\cdot)$ denotes the differential operator. $\mathcal{C N}(\mathbf{a}, \boldsymbol{\Sigma})$ is a complex-valued Gaussian distribution of mean $\mathbf{a}$ and covariance matrix $\boldsymbol{\Sigma}$. I is the identity matrix of appropriate dimension. $\operatorname{det}(\cdot)$ and $\operatorname{Tr}(\cdot)$ stand for the determinant and the trace of a matrix, respectively. $\hat{d}$ is an estimate of the parameter $d .\left\{w_{n}\right\}_{n \in \llbracket 1, N \rrbracket}$ denotes the set of $n$ elements $w_{n}$ with $n \in \llbracket 1, N \rrbracket$, and will often be abbreviated to $\left\{w_{n}\right\}$ in the sequel. $\mathbf{e}_{i}$ is the $i^{\text {th }}$ vector of the canonical basis of appropriate dimension.

\section{BACKGROUND}

\section{A. Problem Formulation}

We assume that $K$ samples $\left\{\mathbf{z}_{k}\right\}_{k \in \llbracket 1, K \rrbracket}$ are available. Each of the data $\mathbf{z}_{k} \in \mathbb{C}^{M}$ corresponds to a realization of a proper circular LR Compound Gaussian process $\mathbf{c}_{k}$ plus an independent additive zero-mean complex $\mathrm{WGN} \mathbf{n}_{k}$ :

$$
\mathbf{z}_{k}=\mathbf{n}_{k}+\mathbf{c}_{k} .
$$

The WGN $\mathbf{n}_{k}$ follows the distribution

$$
\mathbf{n}_{k} \sim \mathcal{C N}\left(\mathbf{0}, \sigma^{2} \mathbf{I}\right),
$$

where the power of the WGN $\sigma^{2}$ is assumed to be known ${ }^{2}$ and fixed to be $\sigma^{2}=1$ without loss of generality. The LR

\footnotetext{
${ }^{2}$ This hypothesis is made for describing a valid theoretical framework. In practice, presented results can be applied with an estimate of $\sigma^{2}$ used as its actual value.
} 
Compound Gaussian [15] $\mathbf{c}_{k}$ is an $M$-dimensional zero-mean complex Gaussian vector (the speckle) with CM $\Sigma$, multiplied by the square root of an independent positive random power factor (the texture) $\tau_{k}$. Each $\mathbf{c}_{k}$ follows then, conditionally to $\tau_{k}$ :

$$
\left(\mathbf{c}_{k} \mid \tau_{k}\right) \sim \mathcal{C N}\left(\mathbf{0}, \tau_{k} \boldsymbol{\Sigma}\right)
$$

where the Compound Gaussian clutter CM $\Sigma$ has a Low Rank structure with $\operatorname{rank}(\boldsymbol{\Sigma})=R<M$. The rank $R$ is assumed to be known and a discussion on this assumption will be provided in the remark below. In this work, we do not assume the knowledge of the texture PDF, and treat each $\tau_{k}$ as an unknown deterministic variable. The likelihood function is then

$$
f\left(\left\{\mathbf{z}_{k}\right\} \mid \boldsymbol{\Sigma},\left\{\tau_{k}\right\}\right)=\prod_{k=1}^{K} \frac{e^{-\mathbf{z}_{k}^{H} \boldsymbol{\Sigma}_{k}^{-1} \mathbf{z}_{k}}}{\pi^{M} \operatorname{det}\left(\boldsymbol{\Sigma}_{k}\right)},
$$

with $\boldsymbol{\Sigma}_{k}=\tau_{k} \boldsymbol{\Sigma}+\mathbf{I}$.

The MLE of the clutter CM $\boldsymbol{\Sigma}$ is therefore defined as the minimizer of the following problem (equivalent to the maximizer of the log-likelihood function):

$$
\begin{array}{cl}
\underset{\boldsymbol{\Sigma}_{k},\left\{\tau_{k}\right\}, \boldsymbol{\Sigma} \succeq \mathbf{0}}{\operatorname{minimize}} & \sum_{k=1}^{K} \log \operatorname{det}\left(\boldsymbol{\Sigma}_{k}\right)+\sum_{k=1}^{K} \mathbf{z}_{k}^{H} \boldsymbol{\Sigma}_{k}^{-1} \mathbf{z}_{k} \\
\text { subject to } & \boldsymbol{\Sigma}_{k}=\tau_{k} \boldsymbol{\Sigma}+\mathbf{I} \\
& \tau_{k} \geq 0 \\
& \operatorname{rank}(\boldsymbol{\Sigma}) \leq R,
\end{array}
$$

where $\Sigma$ is of size $M \times M$. Denote the objective function by $L\left(\boldsymbol{\Sigma},\left\{\tau_{k}\right\}\right)$.

\section{B. Block MM principle}

To solve Problem (A), we adopt the block majorizationminimization (MM) algorithm framework, which is briefly stated below.

Consider the following optimization problem:

$$
\begin{array}{ll}
\underset{\mathbf{x}}{\operatorname{minimize}} & f(\mathbf{x}) \\
\text { subject to } & \mathbf{x} \in \mathcal{X},
\end{array}
$$

where the optimization variable $\mathbf{x}$ can be partitioned into $m$ blocks as $\mathbf{x}=\left(\mathbf{x}^{(1)}, \ldots, \mathbf{x}^{(m)}\right)$, with each $n_{i}$-dimensional block $\mathbf{x}^{(i)} \in \mathcal{X}_{i}$ and $\mathcal{X}=\prod_{i=1}^{m} \mathcal{X}_{i}$.

At the $(t+1)$-th iteration, the $i$-th block $\mathbf{x}^{(i)}$ is updated by solving the following problem:

$$
\begin{array}{ll}
\underset{\mathbf{x}^{(i)}}{\operatorname{minimize}} & g_{i}\left(\mathbf{x}^{(i)} \mid \mathbf{x}_{t}\right) \\
\text { subject to } & \mathbf{x}^{(i)} \in \mathcal{X}_{i},
\end{array}
$$

with $i=(t \bmod m)+1$ (so blocks are updated in cyclic order) and the continuous surrogate function $g_{i}\left(\mathbf{x}^{(i)} \mid \mathbf{x}_{t}\right)$ satisfying the following properties:

$$
\begin{aligned}
f\left(\mathbf{x}_{t}\right) & =g_{i}\left(\mathbf{x}_{t}^{(i)} \mid \mathbf{x}_{t}\right), \\
f\left(\mathbf{x}_{t}^{(1)}, \ldots, \mathbf{x}^{(i)}, \ldots, \mathbf{x}_{t}^{(m)}\right) \leq & g_{i}\left(\mathbf{x}^{(i)} \mid \mathbf{x}_{t}\right) \forall \mathbf{x}^{(i)} \in \mathcal{X}_{i}, \\
f^{\prime}\left(\mathbf{x}_{t} ; \mathbf{d}_{i}^{0}\right)= & g_{i}^{\prime}\left(\mathbf{x}_{t}^{(i)} ; \mathbf{d}_{i} \mid \mathbf{x}_{t}\right) \\
& \forall \mathbf{x}_{t}^{(i)}+\mathbf{d}_{i} \in \mathcal{X}_{i}, \\
& \mathbf{d}_{i}^{0} \triangleq\left(\mathbf{0} ; \ldots ; \mathbf{d}_{i} ; \ldots ; \mathbf{0}\right),
\end{aligned}
$$

where $f^{\prime}(\mathbf{x} ; \mathbf{d})$ stands for the directional derivative at $\mathbf{x}$ along d. In short, at each iteration, the block MM algorithm updates the variables in one block by minimizing a tight upperbound of the function while keeping the value of the other blocks fixed.

In practice, the surrogate functions are usually designed so that each sub-problem (7) can be solved easily, for example in closed-form.

\section{Direct Block MajoriZATION-MinimizATION ALGORITHM}

As rank $(\boldsymbol{\Sigma}) \leq R$, the variable $\boldsymbol{\Sigma}$ can be reparameterized as $\boldsymbol{\Sigma}=\mathbf{W} \mathbf{W}^{H}$ with $\mathbf{W} \in \mathbb{C}^{M \times R}$. Problem (A) can then be written equivalently as

$$
\begin{array}{ll}
\underset{\boldsymbol{\Sigma}_{k},\left\{\tau_{k}\right\}, \mathbf{W}}{\operatorname{minimize}} & \sum_{k=1}^{K} \log \operatorname{det}\left(\boldsymbol{\Sigma}_{k}\right)+\sum_{k=1}^{K} \mathbf{z}_{k}^{H} \boldsymbol{\Sigma}_{k}^{-1} \mathbf{z}_{k} \\
\text { subject to } & \boldsymbol{\Sigma}_{k}=\tau_{k} \mathbf{W} \mathbf{W}^{H}+\mathbf{I} \\
& \tau_{k} \geq 0 .
\end{array}
$$

Following the block MM methodology, we partition the variables as $\left\{\left\{\tau_{k}\right\}, \mathbf{W}\right\}$ and derive an algorithm that updates the blocks in cyclic order (note that variables $\boldsymbol{\Sigma}_{k}$ are implicitly optimized at every iteration while optimizing either $\tau_{k}$ or $\mathbf{W}$ ). In each iteration, an upperbound of the objective function is minimized, which guarantees a monotonic decrement of the objective value.

To be precise, given a starting point $\left\{\left\{\tau_{k}\right\}^{t=0}, \mathbf{W}^{t=0}\right\}$, one iteratively

- updates $\left\{\tau_{k}\right\}^{t+1}$ for fixed $\mathbf{W}=\mathbf{W}^{t}$ by minimizing a set of surrogate functions $L\left(\tau_{k} \mid \tau_{k}^{t}, \mathbf{W}\right)$ for $k \in \llbracket 1, K \rrbracket$,

- updates $\mathbf{W}^{t+1}$ for fixed $\left\{\tau_{k}\right\}=\left\{\tau_{k}\right\}^{t+1}$ by minimizing a surrogate function $L\left(\mathbf{W} \mid\left\{\tau_{k}\right\}, \mathbf{W}^{t}\right)$,

until convergence, which will produce a stationary point of Problem (B). This procedure is summed up in the box Algorithm 1.

\section{A. Update $\left\{\tau_{k}\right\}$ with fixed $\mathbf{W}$}

Let $\mathbf{W}=\mathbf{W}^{t}$ and $\boldsymbol{\Sigma}=\mathbf{W}^{t} \mathbf{W}^{t H}$. To lighten notation, we omit the reference on $t$ for these variables in this part. The objective function is separable in the $\tau_{k}$ 's, and for each of them, the following problem should be solved:

$$
\begin{array}{ll}
\underset{\tau_{k}}{\operatorname{minimize}} & \log \operatorname{det}\left(\tau_{k} \boldsymbol{\Sigma}+\mathbf{I}\right)+\mathbf{z}_{k}^{H}\left(\tau_{k} \boldsymbol{\Sigma}+\mathbf{I}\right)^{-1} \mathbf{z}_{k} \\
\text { subject to } & \tau_{k} \geq 0 .
\end{array}
$$

Eigendecompose $\boldsymbol{\Sigma}$ as $\boldsymbol{\Sigma}=\mathbf{U} \boldsymbol{\Lambda} \mathbf{U}^{T}$, with $\boldsymbol{\Lambda}=$ $\operatorname{diag}\left(\lambda_{1}, \ldots, \lambda_{R}, 0, \ldots, 0\right)$ and $\mathbf{U}=\left[\mathbf{u}_{1}, \ldots, \mathbf{u}_{M}\right]$. The objective function of (B1) can be simplified to

$$
\begin{aligned}
& L\left(\tau_{k} \mid \mathbf{W}\right)=\sum_{m=1}^{M} \log \left(\tau_{k} \lambda_{m}+1\right)+\sum_{m=1}^{M} s_{k m}\left(\tau_{k} \lambda_{m}+1\right)^{-1} \\
& =\sum_{m=1}^{R} \log \left(\tau_{k} \lambda_{m}+1\right)+\sum_{m=1}^{R} s_{k m}\left(\tau_{k} \lambda_{m}+1\right)^{-1}+\text { const. }
\end{aligned}
$$


where $s_{k m}=\left\|\mathbf{z}_{k}^{H} \mathbf{u}_{m}\right\|^{2}$. This function is the sum of quasiconvex functions, which is not necessarily quasi-convex, and has no closed-form minimizer.

Applying the block MM algorithm, we find an upperbound (surrogate function) of $L\left(\tau_{k} \mid \mathbf{W}\right)$, with equality achieved at $\tau_{k}=\tau_{k}^{t}$, and minimize this surrogate function. The update of $\tau_{k}$ is derived based on the following two propositions:

Proposition 1. The function $L\left(\tau_{k} \mid \mathbf{W}\right)$ in (8) can be upperbounded by the surrogate function $L\left(\tau_{k} \mid \tau_{k}^{t}, \mathbf{W}\right)$ defined as

$$
\begin{aligned}
& L\left(\tau_{k} \mid \tau_{k}^{t}, \mathbf{W}\right)=-\beta_{k} \log \tau_{k}+ \\
& \left(\sum_{m=1}^{R} \alpha_{k m}\right) \log \left(\frac{\left(\sum_{m=1}^{R} \frac{\alpha_{k m} \lambda_{m}}{1+\lambda_{m} \tau_{k}^{t}}\right)}{\sum_{m=1}^{R} \alpha_{k m}} \tau_{k}+\frac{\sum_{m=1}^{R} \frac{\alpha_{k m}}{1+\lambda_{m} \tau_{k}^{t}}}{\sum_{m=1}^{R} \alpha_{k m}}\right) \\
& + \text { const., }
\end{aligned}
$$

where

$$
\alpha_{k m}=s_{k m} \frac{\tau_{k}^{t} \lambda_{m}}{\tau_{k}^{t} \lambda_{m}+1}+1
$$

and

$$
\beta_{k}=\sum_{m=1}^{R} s_{k m} \frac{\tau_{k}^{t} \lambda_{m}}{\tau_{k}^{t} \lambda_{m}+1} .
$$

The equality is achieved at $\tau_{k}=\tau_{k}^{t}$.

Proof: See Appendix A.

Proposition 2. The surrogate function $L\left(\tau_{k} \mid \tau_{k}^{t}, \mathbf{W}\right)$ is quasiconvex and has a unique minimizer given by

$$
\tau_{k}^{t+1}=\frac{1}{R} \cdot \frac{\left(\sum_{m=1}^{R} s_{k m} \frac{\tau_{k}^{t} \lambda_{m}}{\tau_{k}^{t} \lambda_{m}+1}\right) \cdot\left(\sum_{m=1}^{R} \frac{\alpha_{k m}}{1+\lambda_{m} \tau_{k}^{t}}\right)}{\sum_{m=1}^{R} \frac{\alpha_{k m} \lambda_{m}}{1+\lambda_{m} \tau_{k}^{t}}} .
$$

\section{Proof: See Appendix B.}

\section{B. Update $\mathbf{W}$ with Fixed $\left\{\tau_{k}\right\}$}

Let now fix $\left\{\tau_{k}\right\}=\left\{\tau_{k}\right\}^{t+1}$. To lighten the notation, we omit the reference on $t$ for this set of variables in this part. To obtain the update of $\mathbf{W}$, we need to solve

$$
\begin{array}{ll}
\underset{\boldsymbol{\Sigma}_{k}, \mathbf{W}}{\operatorname{minimize}} & \sum_{k=1}^{K} \log \operatorname{det}\left(\boldsymbol{\Sigma}_{k}\right)+\sum_{k=1}^{K} \mathbf{z}_{k}^{H} \boldsymbol{\Sigma}_{k}^{-1} \mathbf{z}_{k} \\
\text { subject to } & \boldsymbol{\Sigma}_{k}=\tau_{k} \mathbf{W} \mathbf{W}^{H}+\mathbf{I} .
\end{array}
$$

Without loss of generality assume that $\tau_{k}>0$, otherwise the terms with $\tau_{k}=0$ can be deleted from the summation in the objective function as they are constants.

Problem (B2) has no closed-form minimizer. As in the previous part, we are going to derive a tight upperbound of the objective function $L\left(\mathbf{W} \mid \tau_{k}\right)$, with equality achieved at $\mathbf{W}=\mathbf{W}^{t}$.

Proposition 3. The function

$$
L\left(\mathbf{W} \mid \tau_{k}\right)=\sum_{k=1}^{K} \log \operatorname{det}\left(\boldsymbol{\Sigma}_{k}\right)+\sum_{k=1}^{K} \mathbf{z}_{k}^{H} \boldsymbol{\Sigma}_{k}^{-1} \mathbf{z}_{k},
$$

where $\boldsymbol{\Sigma}_{k}=\tau_{k} \mathbf{W} \mathbf{W}^{H}+\mathbf{I}$, can be upperbounded by the convex quadratic function

$$
L\left(\mathbf{W} \mid \tau_{k}, \mathbf{W}^{t}\right)=\operatorname{Tr}\left(\mathbf{W H} \mathbf{W}^{H}\right)-\operatorname{Tr}\left(\mathbf{L} \mathbf{W}^{H}\right)-\operatorname{Tr}\left(\mathbf{L}^{H} \mathbf{W}\right),
$$

with equality achieved at $\mathbf{W}=\mathbf{W}^{t}$, where the matrices $\mathbf{H}$ and $\mathbf{L}$ are defined according to (41), (42), (46), and (47).

Proof: See Appendix C.

The matrix $\mathbf{H}$ is positive definite by definition, hence the upperbound $L\left(\mathbf{W} \mid \tau_{k}, \mathbf{W}^{t}\right)$ has a unique minimizer given by

$$
\mathbf{W}^{t+1}=\mathbf{L H}^{-1} \text {. }
$$

To efficiently compute this solution, let the SVD of $\mathbf{W}^{t}$ be $\mathbf{W}^{t}=\mathbf{U S V}^{H}$. Then

$$
\mathbf{L}=\sum_{k=1}^{K} \mathbf{L}_{k}^{t}=\left(\sum_{k=1}^{K} \mathbf{z}_{k} \mathbf{z}_{k}^{H} \mathbf{U S}\left(\tau_{k}^{-1} \mathbf{I}+\mathbf{S}^{2}\right)^{-1}\right) \mathbf{V}^{H},
$$

and

$$
\begin{aligned}
\mathbf{H}= & \sum_{k=1}^{K}\left(\left(\mathbf{W}^{t}\right)^{H} \mathbf{W}^{t}+\tau_{k}^{-1} \mathbf{I}\right)^{-1}+\sum_{k=1}^{K} \mathbf{H}_{k}^{t} \\
= & \sum_{k=1}^{K} \mathbf{V}\left(\tau_{k}^{-1} \mathbf{I}+\mathbf{S}^{2}\right)^{-1} \mathbf{S}^{H} \mathbf{U}^{H} \mathbf{z}_{k} \mathbf{z}_{k}^{H} \mathbf{U S}\left(\tau_{k}^{-1} \mathbf{I}+\mathbf{S}^{2}\right)^{-1} \mathbf{V}^{H} \\
& +\sum_{k=1}^{K} \mathbf{V}\left(\tau_{k}^{-1} \mathbf{I}+\mathbf{S}^{2}\right)^{-1} \mathbf{V}^{H}
\end{aligned}
$$

where $\mathbf{S}^{2}=\mathbf{S}^{H} \mathbf{S}$ (since $\mathbf{S}$ is a real diagonal matrix). $\mathbf{W}^{t+1}$ can therefore be computed as

$$
\begin{aligned}
& \mathbf{W}^{t+1}=\left(\sum_{k=1}^{K} \mathbf{z}_{k} \mathbf{z}_{k}^{H} \mathbf{U S}\left(\tau_{k}^{-1} \mathbf{I}+\mathbf{S}^{2}\right)^{-1}\right) \times \\
& \left(\sum _ { k = 1 } ^ { K } \left(\left(\tau_{k}^{-1} \mathbf{I}+\mathbf{S}^{2}\right)^{-1}+\right.\right. \\
& \left.\left.\left(\tau_{k}^{-1} \mathbf{I}+\mathbf{S}^{2}\right)^{-1} \mathbf{S}^{H} \mathbf{U}^{H} \mathbf{z}_{k} \mathbf{z}_{k}^{H} \mathbf{U S}\left(\tau_{k}^{-1} \mathbf{I}+\mathbf{S}^{2}\right)^{-1}\right)\right)^{-1} \mathbf{V}^{H}
\end{aligned}
$$

\section{Convergence Analysis}

Proposition 4. Any limit point of the pair $\left\{\left\{\tau_{k}^{t}\right\}, \mathbf{W}^{t}\right\}$ generated by Algorithm 1 is a stationary point of Problem (B).

Proof: We have proved the quasi-convexity and the uniqueness of the minimizer of the surrogate functions $L\left(\tau_{k} \mid \tau_{k}^{t}, \mathbf{W}^{t}\right)$ and $L\left(\mathbf{W} \mid \tau_{k}, \mathbf{W}^{t}\right)$ in the previous subsections. The algorithm convergence is a direct application of Theorem 2 (a) in [46].

\section{Eigenspace Block Majorization-Minimization ALGORITHM}

As $\operatorname{rank}(\boldsymbol{\Sigma}) \leq R$, the variable $\boldsymbol{\Sigma}$ can be reparameterized by its eigendecomposition:

$$
\boldsymbol{\Sigma}=\sum_{r=1}^{R} c_{r} \mathbf{v}_{r} \mathbf{v}_{r}^{H} .
$$




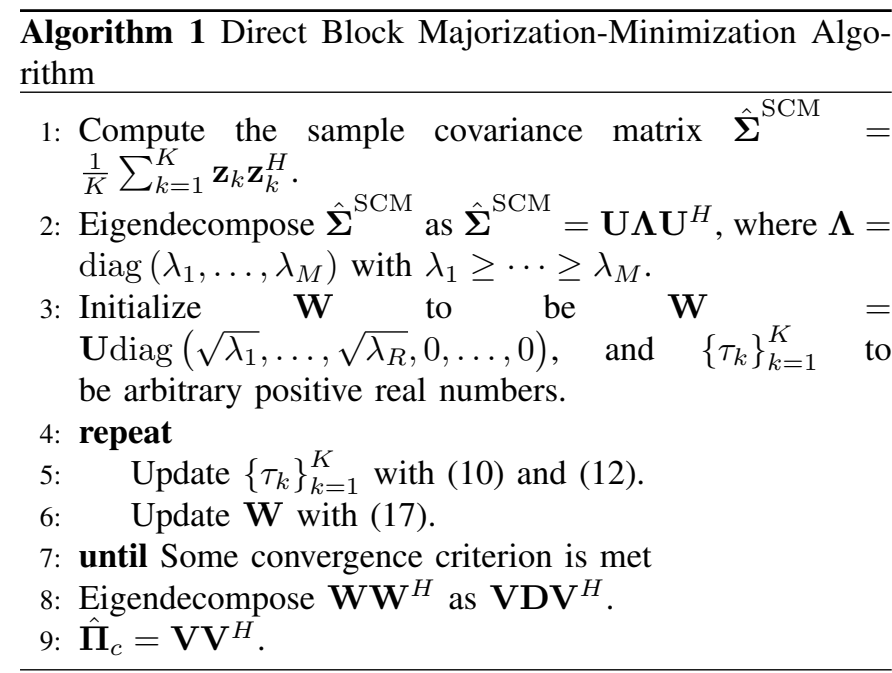

Problem (A) can then be rewritten as

$$
\begin{aligned}
\underset{\left\{\tau_{k}\right\},\left\{c_{r}\right\},\left\{\mathbf{v}_{r}\right\}}{\operatorname{minimize}} & -\sum_{k=1}^{K} \sum_{r=1}^{R} \frac{\tau_{k} c_{r}}{\tau_{k} c_{r}+1} \mathbf{z}_{k}^{H} \mathbf{v}_{r} \mathbf{v}_{r}^{H} \mathbf{z}_{k} \\
& +\sum_{k=1}^{K} \sum_{r=1}^{R} \log \left(1+\tau_{k} c_{r}\right)
\end{aligned}
$$

subject to $\tau_{k} \geq 0, c_{r} \geq 0$, orthonormal $\mathbf{v}_{r}$ 's.

Following the same methodology as in the previous section, we partition the variables as $\left\{\left\{\tau_{k}\right\},\left\{c_{r}\right\},\left\{\mathbf{v}_{r}\right\}\right\}$ and derive an algorithm that updates the blocks in cyclic order by minimizing an upperbound of the objective.

Given a starting point $\left\{\left\{\tau_{k}\right\}^{t=0},\left\{c_{r}\right\}^{t=0},\left\{\mathbf{v}_{r}\right\}^{t=0}\right\}$, one iteratively

- updates $\left\{\tau_{k}\right\}^{t+1}$ for fixed $\left\{c_{r}\right\}=\left\{c_{r}\right\}^{t}$ and $\left\{\mathbf{v}_{r}\right\}=$ $\left\{\mathbf{v}_{r}\right\}^{t}$ by minimizing a set of surrogates functions $L\left(\tau_{k} \mid \tau_{k}^{t},\left\{c_{r}\right\},\left\{\mathbf{v}_{r}\right\}\right)$ for $k \in \llbracket 1, K \rrbracket$,

- updates $\left\{c_{r}\right\}^{t+1}$ for fixed $\left\{\tau_{k}\right\}=\left\{\tau_{k}\right\}^{t+1}$ and $\left\{\mathbf{v}_{r}\right\}=$ $\left\{\mathbf{v}_{r}\right\}^{t}$ by minimizing a set of surrogates functions $L\left(c_{k} \mid\left\{\tau_{k}\right\}, c_{r}^{t},\left\{\mathbf{v}_{r}\right\}\right)$ for $r \in \llbracket 1, R \rrbracket$,

- updates $\left\{\mathbf{v}_{r}\right\}^{t+1}$ for fixed $\left\{\tau_{k}\right\}=\left\{\tau_{k}\right\}^{t+1}$ and $\left\{c_{r}\right\}=\left\{c_{r}\right\}^{t+1}$ by minimizing the objective w.r.t. $\left\{\mathbf{v}_{r}\right\}$ under orthonormality constraints. This step is done by iteratively minimizing surrogate functions $L\left(\left\{\mathbf{v}_{r}\right\} \mid\left\{\tau_{k}\right\}\left\{c_{r}\right\},\left\{\mathbf{v}_{r}\right\}^{t}\right)$ (so there is an inner-loop in the block MM iterations).

This procedure is summed up in the box Algorithm 2.

\section{A. Update $\left\{\tau_{k}\right\}$ with Fixed $\left\{c_{r}\right\}$ and $\left\{\mathbf{v}_{r}\right\}$}

Let $\left\{c_{r}\right\}=\left\{c_{r}\right\}^{t}$ and $\left\{\mathbf{v}_{r}\right\}=\left\{\mathbf{v}_{r}\right\}^{t}$. To lighten notation, we omit the reference on $t$ for these variables in this part. The objective function is separable in the $\tau_{k}$ 's, and for each of them the following problem (noticing that $\boldsymbol{\Sigma}$ has $M-R$ zero eigenvalues) should be solved:

$$
\begin{array}{ll}
\underset{\tau_{k}}{\operatorname{minimize}} & \sum_{r=1}^{R} \log \left(1+\tau_{k} c_{r}\right)+\sum_{r=1}^{R} \frac{s_{k r}}{1+\tau_{k} c_{r}} \\
\text { subject to } & \tau_{k} \geq 0,
\end{array}
$$

with $s_{k r}=\left\|\mathbf{z}_{k}^{H} \mathbf{v}_{r}\right\|^{2}$. Notice that the objective function of (C1) has the same form as (8), therefore we can apply Propositions 1 and 2 to obtain the $\tau_{k}$ 's updates as

$$
\tau_{k}^{t+1}=\frac{1}{R} \cdot \frac{\left(\sum_{r=1}^{R} s_{k r} \frac{\tau_{k}^{t} c_{r}}{\tau_{k}^{t} c_{r}+1}\right) \cdot\left(\sum_{r=1}^{R} \frac{\alpha_{k r}}{1+c_{r} \tau_{k}^{t}}\right)}{\sum_{r=1}^{R} \frac{\alpha_{k r} c_{r}}{1+c_{r} \tau_{k}^{t}}}
$$

where $\alpha_{k r}=s_{k r} \frac{\tau_{k}^{t} c_{r}}{\tau_{k}^{t} c_{r}+1}+1$.

\section{B. Update $\left\{c_{r}\right\}$ with Fixed $\left\{\tau_{k}\right\}$ and $\left\{\mathbf{v}_{r}\right\}$}

Let $\left\{\tau_{k}\right\}=\left\{\tau_{k}\right\}^{t+1}$ and $\left\{\mathbf{v}_{r}\right\}=\left\{\mathbf{v}_{r}\right\}^{t}$. As before we omit the reference on $t$ for these variables in this part. The objective function is separable in the $c_{r}$ 's, and for each of them the following problem should be solved:

$$
\begin{array}{ll}
\underset{c_{r}}{\operatorname{minimize}} & \sum_{k=1}^{K} \log \left(1+\tau_{k} c_{r}\right)+\sum_{k=1}^{K} \frac{s_{k r}}{1+\tau_{k} c_{r}} \\
\text { subject to } & c_{r} \geq 0,
\end{array}
$$

with $s_{k r}=\left\|\mathbf{z}_{k}^{H} \mathbf{v}_{r}\right\|^{2}$. Notice that the $c_{r}$ 's in (C2) play a similar role as the $\tau_{k}$ 's in (C1). Similar to the update of $\tau_{k}$, we can apply Propositions 1 and 2 to obtain the $c_{r}$ 's updates as

$$
c_{r}^{t+1}=\frac{1}{K} \cdot \frac{\left(\sum_{k=1}^{K} s_{k r} \frac{\tau_{k} c_{r}^{t}}{\tau_{k} c_{r}^{t}+1}\right) \cdot\left(\sum_{k=1}^{K} \frac{\alpha_{k r}}{1+c_{r}^{t} \tau_{k}}\right)}{\sum_{k=1}^{K} \frac{\alpha_{k r} \tau_{k}}{1+c_{r}^{t} \tau_{k}}}
$$

where $\alpha_{k r}=s_{k r} \frac{\tau_{k} c_{r}^{t}}{\tau_{k} c_{r}^{t}+1}+1$.

\section{Update $\left\{\mathbf{v}_{r}\right\}$ with Fixed $\left\{\tau_{k}\right\}$ and $\left\{c_{r}\right\}$}

With $\left\{\tau_{k}\right\}$ and $\left\{c_{r}\right\}$ fixed, minimizing the objective w.r.t. $\left\{\mathbf{v}_{r}\right\}$ is equivalent to solving the problem

$$
\begin{array}{ll}
\underset{\left\{\mathbf{v}_{r}\right\}}{\operatorname{maximize}} & \sum_{r=1}^{R} \mathbf{v}_{r}^{H} \mathbf{M}_{r} \mathbf{v}_{r} \\
\text { subject to } & \text { orthonormal } \mathbf{v}_{r} \text { 's, }
\end{array}
$$

where

$$
\mathbf{M}_{r}=\sum_{k=1}^{K} \frac{\tau_{k} c_{r}}{\tau_{k} c_{r}+1} \mathbf{z}_{k} \mathbf{z}_{k}^{H} .
$$

We start with the following proposition.

Proposition 5. The function

$$
L\left(\left\{\mathbf{v}_{r}\right\} \mid\left\{\tau_{k}\right\},\left\{c_{r}\right\}\right)=\sum_{r=1}^{R} \mathbf{v}_{r}^{H} \mathbf{M}_{r} \mathbf{v}_{r}
$$

can be lowerbounded by the surrogate function

$$
\begin{aligned}
& L\left(\left\{\mathbf{v}_{r}\right\} \mid\left\{\tau_{k}\right\},\left\{c_{r}\right\},\left\{\mathbf{v}_{r}\right\}^{t}\right) \\
& =\sum_{r=1}^{R}\left[\left(\mathbf{v}_{r}^{t}\right)^{H} \mathbf{M}_{r} \mathbf{v}_{r}+\mathbf{v}_{r}^{H} \mathbf{M}_{r} \mathbf{v}_{r}^{t}\right]+\text { const. }
\end{aligned}
$$

with equality achieved at $\left\{\mathbf{v}_{r}\right\}=\left\{\mathbf{v}_{r}\right\}^{t}$.

Proof: As the matrices $\mathbf{M}_{r}$ are Hermitian positive semidefinite, the objective function is convex, and therefore 


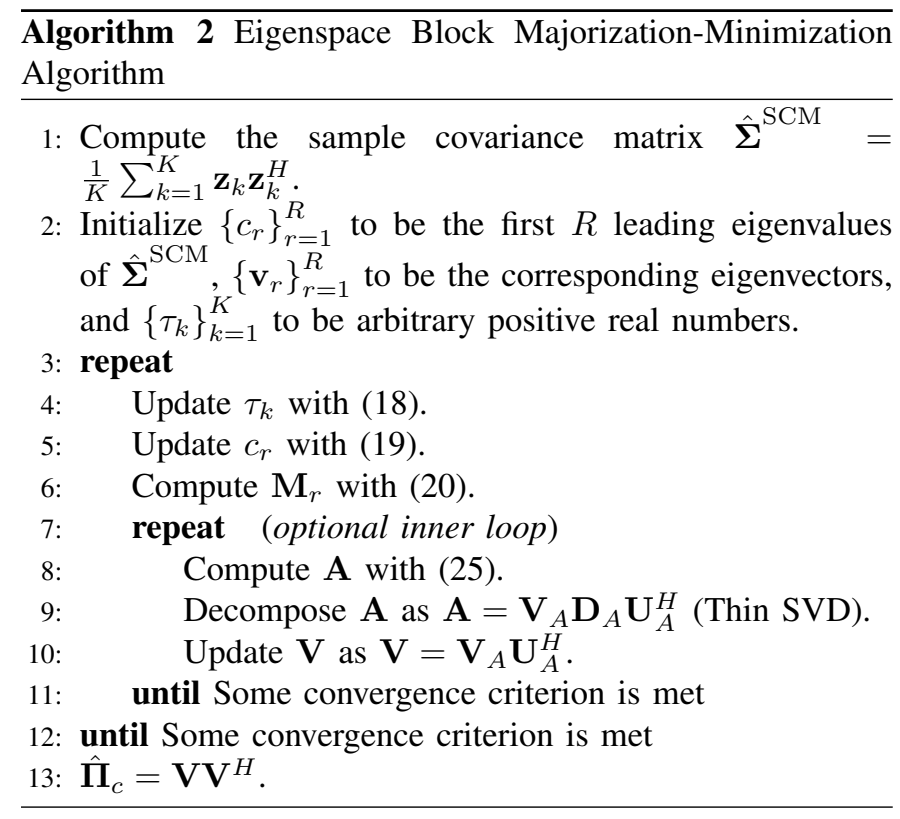

is minorized by its first order Taylor expansion at $\mathbf{v}_{r}^{t}$, which is the considered surrogate function (22).

Maximizing $L\left(\left\{\mathbf{v}_{r}\right\} \mid\left\{\tau_{k}\right\},\left\{c_{r}\right\},\left\{\mathbf{v}_{r}\right\}^{t}\right)$, under orthonormality constraints on the $\left\{\mathbf{v}_{r}\right\}$, is equivalent to solving

$$
\begin{array}{ll}
\underset{\mathbf{V}}{\operatorname{maximize}} & \operatorname{Tr}\left(\mathbf{A}^{H} \mathbf{V}\right)+\operatorname{Tr}\left(\mathbf{V}^{H} \mathbf{A}\right) \\
\text { subject to } & \mathbf{V}^{H} \mathbf{V}=\mathbf{I},
\end{array}
$$

where

$$
\mathbf{V}=\left[\mathbf{v}_{1}, \ldots, \mathbf{v}_{R}\right]
$$

and

$$
\mathbf{A}=\left[\mathbf{M}_{1} \mathbf{v}_{1}^{t} ; \ldots ; \mathbf{M}_{R} \mathbf{v}_{R}^{t}\right]
$$

i.e., $\mathbf{A}$ is constructed by stacking the column vectors $\mathbf{M}_{r} \mathbf{v}_{r}^{t}$. Problem (23) is equivalent to

$$
\begin{array}{ll}
\underset{\mathbf{V}}{\operatorname{minimize}} & \|\mathbf{A}-\mathbf{V}\|_{F}^{2} \\
\text { subject to } & \mathbf{V}^{H} \mathbf{V}=\mathbf{I} .
\end{array}
$$

Let the thin SVD of $\mathbf{A}$ be $\mathbf{A}=\mathbf{V}_{A} \mathbf{D}_{A} \mathbf{U}_{A}^{H}$, the optimal $\mathbf{V}$ is given by [45]

$$
\mathbf{V}^{t+1}=\mathbf{V}_{A} \mathbf{U}_{A}^{H}
$$

Note that in this step, we maximize the objective function of (C3) by iteratively maximizing a series of surrogates (steps 7-11 in Algorithm 2). These iterations lead to a local solution of (C3). While the block MM framework indicates a cyclic update of the variables $\left\{\tau_{k}\right\},\left\{c_{r}\right\},\left\{\mathbf{v}_{r}\right\}$, our numerical tests reveal that including this inner loop provides a faster decreasing rate of the objective value than updating the $\mathbf{v}_{r}$ 's only once.

\section{Convergence Analysis}

Since the constraint set of Problem (C) is non-convex, the convergence result provided in [46] cannot be applied here. To the best of our knowledge, there is no convergence result of general block descent type algorithms with a non-convex constraint set. The sequence of objective values generated by Algorithm 2 will converge because of monotonicity, but the convergence of the points $\left(\left\{\tau_{k}\right\}^{t},\left\{c_{r}\right\}^{t},\left\{\mathbf{v}_{r}\right\}^{t}\right)$ remains unknown. Nevertheless, section VI will show that the numerical performance of Algorithm 2 is satisfactory.

\section{HYPER-PARAMETERS ESTIMATION}

The clutter subspace estimation algorithms proposed in this paper assume the prior knowledge of two hyper-parameters: the clutter rank $R$ and the white Gaussian noise power $\sigma^{2}$. These assumptions are made for deriving a valid and clear theoretical framework, focusing on the core of the subspace estimation problem. In practice, the proposed algorithms can be called using plug-in estimates $\hat{R}$ and $\hat{\sigma}^{2}$.

To estimate the clutter rank, there are several methods present in the literature (e.g., [8]-[10] and references therein). Moreover, in some applications, the clutter rank can be derived from the geometry of the system (such as in STAP [11] thanks to the Brennan rule [12]).

Regarding the WGN power, the algorithms derived in this paper can also be extended to estimate it by introducing one more block for the parameter $\sigma^{2}$. The update of this block $\sigma^{2}$ for $\left\{\boldsymbol{\Sigma}_{k}\right\}$ fixed can be obtained by majorizing the objective function w.r.t. $\sigma^{2}$ in a way similar to the parameters $\left\{\tau_{k}\right\}$. To account for possible knowledge on the lower and upper bounds of the WGN power [47], [48] (denoted by $\sigma_{l}^{2}$ and $\sigma_{u}^{2}$, respectively), one can further impose the constraint that $\sigma_{l}^{2} \leq \sigma^{2} \leq \sigma_{u}^{2}$. For the sake of simplicity and presentation clarity, we focused on the part of estimating $\left\{\boldsymbol{\Sigma}_{k}\right\}$, which is the bottleneck of the considered problem. Estimating $\sigma^{2}$ jointly is therefore left as a potential extension of this work.

\section{NUMERICAL RESUlTS}

This section is devoted to numerical simulations to illustrate the performance of different CM estimators in the considered context. In particular, we will study the following clutter subspace projector estimators:

- $\hat{\boldsymbol{\Pi}}_{S C M}$ : the clutter subspace projector estimator derived from the EVD of the SCM defined as $\hat{\boldsymbol{\Sigma}}_{S C M}=$ $\sum_{k=1}^{K} \mathbf{z}_{k} \mathbf{z}_{k}^{H} / K$.

- $\hat{\Pi}_{S F P E}$ : the clutter subspace projector estimator derived from the EVD of the Shrinkage-FPE (SFPE) [34]-[36], which is a regularized Tyler's estimator [24] defined as the unique solution of the following fixed-point equation:

$\hat{\mathbf{\Sigma}}_{S-F P E}(\beta)=\frac{(1-\beta) M}{K} \sum_{k=1}^{K} \frac{\mathbf{z}_{k} \mathbf{z}_{k}^{H}}{\mathbf{z}_{k}^{H} \hat{\mathbf{\Sigma}}_{S-F P E}^{-1}(\beta) \mathbf{z}_{k}}+\beta \mathbf{I}$,

for $\beta \in(\max (0,1-K / M), 1]$. This estimator can be computed with the simple fixed point iterations provided in [34]-[36]. Since there is no rule to adaptively 
select the optimal shrinkage parameter $\beta$ for the considered problem, we test the following values: $\beta_{1}=$ $\max (1-K / M+\epsilon, 0)$, which is the lowest $\beta$ allowed for the under-sampled cases $(K \leq M)$ and coincides with Tyler's estimator (referred to as FPE) for the oversampled cases $(K>M) ; \beta_{2}=\left(\beta_{1}+\beta_{3}\right) / 2$; and $\beta_{3}=1-\epsilon$. We set $\epsilon=10^{-2}$.

- $\hat{\boldsymbol{\Pi}}_{M L E-M M 1}$ : the clutter subspace projector MLE computed with the direct block-MM Algorithm in Section III

- $\hat{\boldsymbol{\Pi}}_{M L E-M M 2}$ : the clutter subspace projector MLE computed with the Eigenspace block-MM Algorithm in Section IV.

- $\hat{\boldsymbol{\Pi}}_{M L E}$ : the clutter subspace projector MLE under high CNR assumption, computed with Algorithm 1 provided in reference [44]. This algorithm will be referred to as "Algorithm 3" in the rest of the paper.

- $\hat{\Pi}_{A-M L E}$ : the clutter subspace projector Approached MLE under high CNR assumption, computed with Algorithm 2 provided in reference [44]. This algorithm will be referred to as "Algorithm 4" in the rest of the paper.

\section{A. Validation Simulations and Algorithm Complexity}

Simulation parameters: Samples $\mathbf{z}_{k}$ are generated according to the LR Compound Gaussian plus WGN model described in section II: $\mathbf{z}_{k}=\mathbf{c}_{k}+\mathbf{n}_{k}$. The WGN is distributed as $\mathbf{n}_{k} \sim \mathcal{C N}\left(\mathbf{0}, \sigma^{2} \mathbf{I}\right)$ and $\sigma^{2}=1$. The LR Compound Gaussian clutter is distributed as $\left(\mathbf{c}_{k} \mid \tau_{k}\right) \sim \mathcal{C N}\left(\mathbf{0}, \tau_{k} \boldsymbol{\Sigma}\right)$, with a random texture $\tau_{k}$, i.i.d. generated for each sample. The texture PDF is a Gamma distribution (leading to a $\mathrm{K}$ distributed clutter) of shape parameter $\nu$ and scale parameter $1 / \nu$, denoted $\tau \sim \Gamma(\nu, 1 / \nu)$, which satisfies $\mathbb{E}(\tau)=1$. The rank $R$ clutter $\mathrm{CM} \boldsymbol{\Sigma}_{c}$ is constructed with the largest $R$ eigenvalues and the corresponding eigenvectors of a Toeplitz matrix of correlation parameter $\rho \in[0,1]$. This matrix is then scaled to set the CNR, defined as $\mathrm{CNR}=\mathbb{E}(\tau) \operatorname{Tr}(\boldsymbol{\Sigma}) /\left(R \sigma^{2}\right)$, to a given value.

Fig. 1 displays a typical realization of the objective value versus the number of iterations of different algorithms. The objective value at each inner loop is also displayed for the proposed algorithms. One can observe that the MLE and AMLE algorithms from [44] converge to a sub-optimal point of the problem. This was to be expected since these algorithms are optimizing a modified likelihood (assuming High CNR, the WGN is ignored over the clutter subspace). The MLE Algorithm provides a slightly better objective value than the AMLE algorithm, thanks to the use of the modified gradient descent algorithm [45] instead of an EVD relaxation for updating the subspace estimate. Contrary to these algorithms, the Block MM algorithm (Algorithm 1) converges to a critical point with a smaller objective value. We also notice that Algorithm 2 converges in practice to the same point as Algorithm 1, i.e., a critical point.

Fig. 2 displays a typical another realization of the objective value versus the time of computation. It illustrates that despite a fast convergence, Algorithm 3 has a slow computation due to the use of the modified gradient descent [45]. It also shows that
Algorithm 2 is less computationally intensive than Algorithm 1 since it requires less time to converge. This can be explained by the fact that constructing the update of $\mathbf{W}$ (step 6) in Algorithm 1 has a complexity that grows linearly with the sample size. On the contrary, the inner loops (steps 7-11) in Algorithm 2 involve the SVD of a matrix of fixed dimension, which is not costly in comparison.

Fig. 3 displays the mean NMSE criterion $\left(\mathbb{E}\left(\| \hat{\boldsymbol{\Pi}}_{c}-\right.\right.$ $\left.\boldsymbol{\Pi}_{c} \|^{2}\right) / R$ for a given estimator $\hat{\boldsymbol{\Pi}}_{c}$ ) versus $K$ of the estimators $\hat{\boldsymbol{\Pi}}_{S C M}, \hat{\boldsymbol{\Pi}}_{S F P E}$ (with $\beta_{1}, \beta_{2}$ and $\beta_{3}$ ), $\hat{\boldsymbol{\Pi}}_{M L E-M M 1}$, $\hat{\boldsymbol{\Pi}}_{M L E-M M 2}, \hat{\boldsymbol{\Pi}}_{M L E}$ and $\hat{\boldsymbol{\Pi}}_{A-M L E}$ for a given configuration (computed over 100 Monte-Carlo simulations). It illustrates the performance of the proposed methods: block MM Algorithms 1 and 2 reach the identically lowest NMSE, and algorithms from [44] lead to slightly higher NMSE, yet better than the SFPE with various $\beta$, and the SCM.

Fig. 4 displays the mean computation time (over 100 Monte Carlo simulations) of the algorithms for the same set of parameters as in previous figures. The stop criterion for each algorithm is when the minimum achievable objective value is reached up to $1 \%$ (The limit value is obtained by a prior run of the considered algorithm for a sufficiently long time until the objective value stabilizes). This criterion is chosen to provide a fair comparison in terms of computational time for algorithms with different convergence rates. The computation time of MLE Algorithm 3 is not displayed since this algorithm (relying on a costly gradient descent) always reaches a maximum time stop criterion, greatly larger than the presented values (as seen in Fig. 2). One can observe that Algorithm 2 and 4 are the "fastest" algorithms, which is due to their low computational cost at each step. Algorithm 4 is the less computationally intensive since it has a fast convergence, as observed in Fig. 1 and Fig. 2. Nevertheless, Algorithm 1 and 2 offer better performance (see Fig. 3). We also point out that, although Algorithm 2 is computationally faster than Algorithm 1, the latter has more theoretical guarantees (convergence to a critical point). Both Fig. 3 and Fig. 4 illustrate the applicative interest of the two proposed computation methods. Indeed, they reach the best estimation performance for the considered model at a low computational cost.

\section{B. Application to LR-STAP filtering}

STAP is a technique used in airborne phased array radar to detect a moving target embedded in an interference background such as jamming or strong clutter [11]. The radar receiver consists of an array of $Q$ antenna elements processing $P$ pulses in a coherent processing interval. The received signal is $\mathbf{z}=\alpha \mathbf{p}+\mathbf{c}+\mathbf{n}$, where $\alpha$ is the target power and $\mathbf{p}$ is the so-called STAP steering vector, $\mathbf{c}$ is the heterogeneous ground clutter and $\mathbf{n}$ is the thermal noise. It is important to notice that the application fits the model considered in this paper since in side looking STAP, the clutter CM is known to be LR. Moreover, the rank of the clutter CM can be evaluated thanks to the Brennan Rule [12]. Since the clutter could behave heterogeneously, the total interference can be modeled as LR Compound Gaussian (the ground clutter) plus WGN (the thermal noise). 


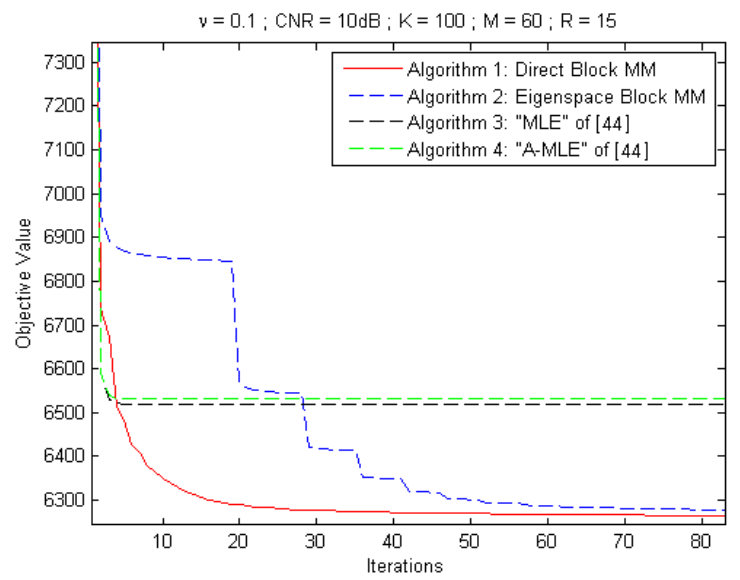

Figure 1. Objective value versus number of iterations for different MLE algorithms: Direct Block-MM (red), Eigenspace Block-MM (blue), "MLE" algorithm of [44] (black), "A-MLE" algorithm of [44] (green). $K=100$, $R=15, \nu=0.1, \rho=0.9, \mathrm{CNR}=10 \mathrm{~dB}$.

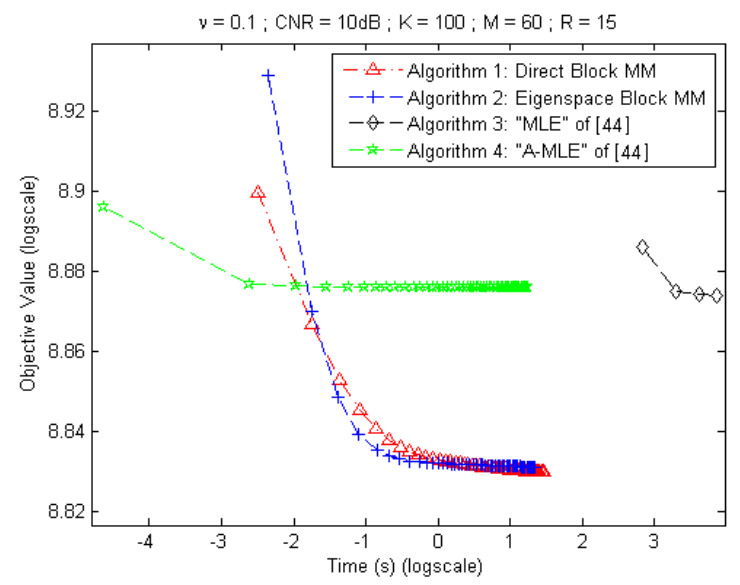

Figure 2. Objective value versus computation time for different MLE algorithms: Direct Block-MM (red), Eigenspace Block-MM (blue), "MLE" algorithm of [44] (black), "A-MLE" algorithm of [44] (green). $K=100$, $R=15, \nu=0.1, \rho=0.9, \mathrm{CNR}=10 \mathrm{~dB}$.

The theoretical optimal STAP filter is $\mathbf{w}_{\text {opt }}=\boldsymbol{\Sigma}_{t o t}^{-1} \mathbf{p}$ [11]. In the context of an LR clutter, it is well-known that a correct sub-optimal filter [6], [14] is $\mathbf{w}_{l r}=\boldsymbol{\Pi}_{c}^{\perp} \mathbf{p}=\left(\mathbf{I}-\boldsymbol{\Pi}_{c}\right) \mathbf{p}$. In practice, $\Pi_{c}^{\perp}$ is unknown and has to be estimated using the samples $\left\{\mathbf{z}_{k}\right\}$ to perform adaptive filtering. The adaptive LR filter is then $\hat{\mathbf{w}}_{l r}=\hat{\boldsymbol{\Pi}}_{c}^{\perp} \mathbf{d}=\left(\mathbf{I}-\hat{\boldsymbol{\Pi}}_{c}\right) \mathbf{p}$, with $\hat{\boldsymbol{\Pi}}_{c}$ being an estimate of the clutter subspace projector. Consequently, the performance of the LR filters directly relies on the estimation accuracy of $\Pi_{c}$. To evaluate the performance, we use the SINR-Loss criterion [11], which is the expected ratio between the $S I N R_{\text {out }}$ computed for $\hat{\mathbf{w}}_{l r}$, and $S I N R_{\max }$ computed for the optimal filter $\mathbf{w}=\boldsymbol{\Sigma}_{t o t}^{-1} \mathbf{d}$. For an estimate of the clutter subspace $\hat{\boldsymbol{\Pi}}_{c}$, the SINR-Loss expression is given by

$$
\begin{aligned}
\rho_{\hat{\mathbf{\Pi}}_{c}} & =\frac{S I N R_{\text {out }}}{S I N R_{\max }} \\
& =\mathbb{E}\left(\frac{\left(\mathbf{p}^{H} \hat{\boldsymbol{\Pi}}_{c}^{\perp} \mathbf{p}\right)^{2}}{\left(\mathbf{p}^{H} \hat{\boldsymbol{\Pi}}_{c}^{\perp} \boldsymbol{\Sigma}_{t o t} \hat{\boldsymbol{\Pi}}_{c}^{\perp} \mathbf{p}\right)\left(\mathbf{p}^{H} \boldsymbol{\Sigma}_{\text {tot }}^{-1} \mathbf{p}\right)}\right) .
\end{aligned}
$$

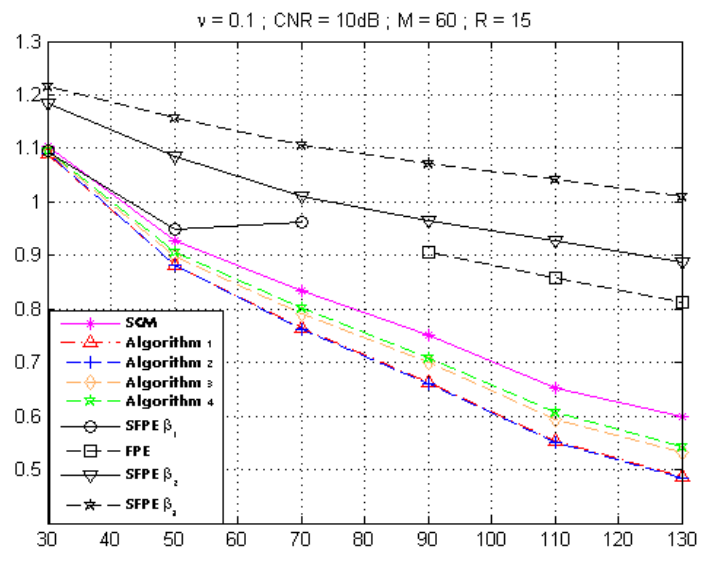

Figure 3. Mean NMSE of the estimators $\hat{\boldsymbol{\Pi}}_{S C M_{\hat{\lambda}}}$ (magenta), $\hat{\boldsymbol{\Pi}}_{M L E}$ (orange), $\hat{\boldsymbol{\Pi}}_{A-M L E}$ (green), $\hat{\boldsymbol{\Pi}}_{M L E-M M 1}$ (red), $\hat{\boldsymbol{\Pi}}_{M L E-M M 2}$ (blue), $\hat{\boldsymbol{\Pi}}_{S F P E}$ for different $\beta$ (black), versus the number of samples $K . M=60$, $R=15, \nu=0.1, \rho=0.9, \mathrm{CNR}=10 \mathrm{~dB}$.

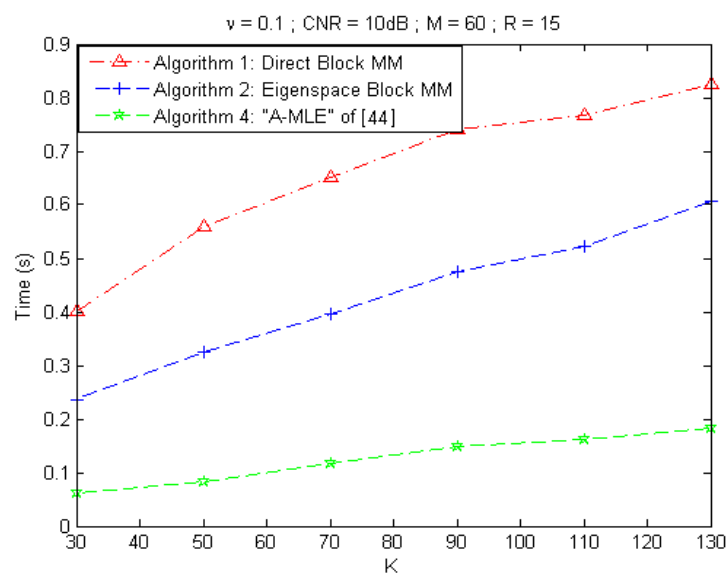

Figure 4. Mean Computation time of each MLE algorithm over 100 MonteCarlo Simulation, versus $K$. Same parameters as Fig. 3. Algorithms stop criterion: minimum achievable objective value is reached up to $1 \%$. Stopping criterion for Algorithm 2 inner loop: $\mathbf{V}: \frac{\left\|\mathbf{V}^{t+1}-\mathbf{V}^{t}\right\|_{F}^{2}}{M \times R} \leq 10^{-6}$. Computer specifics: Intel(R) Core(TM) i5-3230M CPU @ $2.6 \mathrm{Ghz}$.

We consider the following STAP configuration. The number $Q$ of sensors is 8 and the number $P$ of coherent pulses is also 8 . The center frequency and the bandwidth are equal to $f_{0}=450 \mathrm{MHz}$ and $B=4 \mathrm{MHz}$, respectively. The radar velocity is $100 \mathrm{~m} / \mathrm{s}$. The inter-element spacing is $d=\frac{c}{2 f_{0}}(c$ is the celerity of light) and the pulse repetition frequency is $f_{r}=600 \mathrm{~Hz}$. The clutter $\mathrm{CM}$ is computed according to the model described in [11]. The rank of the clutter CM $\boldsymbol{\Sigma}$ is evaluated from the Brennan rule and is equal to $R=15 \ll 64$, therefore, the LR assumption is valid. The texture PDF is a Gamma distribution of shape parameter $\nu=0.1$ and scale parameter $1 / \nu$, so the clutter follows a K-distribution. The target $\mathbf{p}$ has a celerity of $V=35 \mathrm{~m} / \mathrm{s}$ and is at $+10^{\circ}$ Azimuth. CNR is defined as $\mathrm{CNR}=\mathbb{E}(\tau) \operatorname{Tr}\left(\boldsymbol{\Sigma}_{c}\right) /\left(\sigma^{2} R\right)$, and we set $\sigma^{2}=1$. 
Since the two proposed algorithms have been shown to reach identical performance in Fig. 3, we only display the results for the projector estimate computed with the fastest algorithm, namely Algorithm 2 (Eigenspace Block MM), referred to as $\hat{\boldsymbol{\Pi}}_{E B M M}$ in the following. Algorithm 3 and Algorithm 4 (respectively "MLE" and "A-MLE" of [44]) have also been shown to reach identical performance, therefore we only display results for the projector estimate computed with Algorithm 3, referred to as $\hat{\boldsymbol{\Pi}}_{A-M L E}$.

Fig. 5 displays the SINR-Loss versus $K$ for various clutter configurations: from average to high CNR $(10 \mathrm{~dB}, 20 \mathrm{~dB}$, and $30 \mathrm{~dB})$ and for mildly $(\nu=1)$ and highly $(\nu=0.1)$ heterogeneous clutter. First, one can state that $\hat{\boldsymbol{\Pi}}_{E B M M}$ reaches the best SINR-Loss for all configurations. Under standard conditions ( $20 \mathrm{~dB}$ and $30 \mathrm{~dB}$ of CNR and $\nu=1), \hat{\Pi}_{S C M}$ and $\hat{\boldsymbol{\Pi}}_{A-M L E}$ provide a clutter subspace projector estimate that is close to the $\hat{\boldsymbol{\Pi}}_{E B M M}$, hence they have similar performances in terms of SINR-Loss. One can observe that under these conditions, the classical $-3 \mathrm{~dB}$ SINR-Loss of the filter built from $\hat{\boldsymbol{\Pi}}_{S C M}$ is reached with $K \simeq 2 R$ samples, as theoretically expected from [14], [22]. $\hat{\boldsymbol{\Pi}}_{F P E}$ also reaches a performance close to $\hat{\boldsymbol{\Pi}}_{M L E}$ but requires $K>M$ samples to be computed. $\hat{\boldsymbol{\Pi}}_{S F P E}$ can be computed with a smaller $K$, but its SINR-Loss decreases as $\beta$ increases (which is expected since a larger $\beta$ implies a higher bias). When standard conditions are not met (low/average CNR or highly heterogeneous clutter), one can see that the performance of $\hat{\boldsymbol{\Pi}}_{F P E}$ and $\hat{\boldsymbol{\Pi}}_{S F P E}$ greatly drops compared to other estimators. On the contrary, $\hat{\boldsymbol{\Pi}}_{E B M M}$ offers a better resistance to these conditions and also outperforms $\hat{\boldsymbol{\Pi}}_{S C M}$ and $\hat{\boldsymbol{\Pi}}_{A-M L E}$.

\section{Note on the robustness to a wrong rank evaluation}

One can not directly study the estimation accuracy (NMSE) on $\Pi_{c}$ for estimators with various ranks since projectors on subspaces of different dimension are not comparable objects. Such a comparison in NMSE can be done, for example, in terms of reconstructed total CM. For this criterion, [44] showed that the proposed approach is robust to a misevaluated rank.

The robustness to a wrong rank evaluation can also be relevantly studied through a criterion linked to the considered application. For example, for the LR-filtering problem:

- If the rank is under-evaluated, then a portion of the interference may not be completely canceled by the filter, leading to poor performances in terms of output SINR.

- If the rank is over-evaluated, the interference may be completely canceled, but the filter can also cancel a part of the target response, also leading to a lower output SINR.

However, these phenomena depend highly on the clutter and target parameters, which is why a corresponding study goes beyond the scope of this paper, as it is more a problem related to a process design rather than a subspace estimation accuracy.

\section{CONCLUSIONS}

In this paper, we derived two algorithms based on the block MM framework for computing the MLE of the CM parameter when the samples are modeled as the sum of an LR Compound Gaussian (with known rank) and a WGN. This complex problem was initially considered in [43] and [44], where several algorithms were proposed. The new algorithms proposed in this paper enjoy two major advantages: firstly, they do not rely on any heuristic (as in [43]) or high CNR assumption (as in [44]), thus they reach the exact MLE (at least locally) of the considered problem with no approximation; secondly, they are computationally faster and easier to implement than the ones derived in [43] and [44], as they do not require the use of the modified gradient descent algorithm [45], which can be computationally expensive. The performance of the related estimators was illustrated on Space Time Adaptive Processing filtering for airborne radar simulations.

As a side benefit of this study, we emphasize that the proposed algorithms allow a full estimation of the CM parameters, while presented simulations only focus on the clutter subspace projector estimation. They could therefore be suitable for other applications that involve the estimation of Low Rank structured Covariance Matrices.

\section{APPENDIX A}

PROOF OF PROPOSITION 1

Before going to the formal proof of Proposition 1, we need to first state the two following lemmas:

Lemma 6 (Eq.(5) of [49]). For $x_{i}>0$ and $\alpha_{i} \in \mathbb{R}$, the function $\prod_{i=1}^{n} x_{i}^{\alpha_{i}}$ can be lower bounded by

$$
\prod_{i=1}^{n} x_{i}^{\alpha_{i}} \geq \prod_{j=1}^{n}\left(x_{j}^{t}\right)^{\alpha_{j}}\left(1+\sum_{i=1}^{n} \alpha_{i} \log x_{i}-\sum_{i=1}^{n} \alpha_{i} \log x_{i}^{t}\right),
$$

where $x_{i}^{t}$ is some arbitrary positive real number. The equality is achieved at $x_{i}=x_{i}^{t}$. More specifically, in the unidimensional case $n=1$ and for $\alpha_{1}=1$, one has

$$
x \geq x^{t}\left(1+\log (x)-\log \left(x^{t}\right)\right)
$$

Lemma 7. The function $\sum_{i=1}^{n} \alpha_{i} \log \left(1+c_{i} x\right)$ with $\alpha_{i}>0$ can be upperbounded by

$$
\begin{aligned}
\sum_{i=1}^{n} \alpha_{i} \log \left(1+c_{i} x\right) & \leq \sum_{i=1}^{n} \alpha_{i} \log \left(1+c_{i} x^{t}\right)+ \\
& \left(\sum_{i=1}^{n} \alpha_{i}\right) \log \left(\frac{\sum_{i=1}^{n} \alpha_{i} \cdot \frac{1+c_{i} x}{1+c_{i} x^{t}}}{\sum_{i=1}^{n} \alpha_{i}}\right),
\end{aligned}
$$

where $x^{t}$ is some arbitrary real number such that $1+c_{i} x^{t}>0$, $\forall i$. The equality is achieved at $x=x^{t}$

Proof: The $\log (\cdot)$ function is concave, and Jensen's inequality states that

$$
\log \left(\frac{\sum_{i=1}^{n} \alpha_{i} z_{i}}{\sum_{i=1}^{n} \alpha_{i}}\right) \geq \frac{\sum_{i=1}^{n} \alpha_{i} \log z_{i}}{\sum_{i=1}^{n} \alpha_{i}}
$$



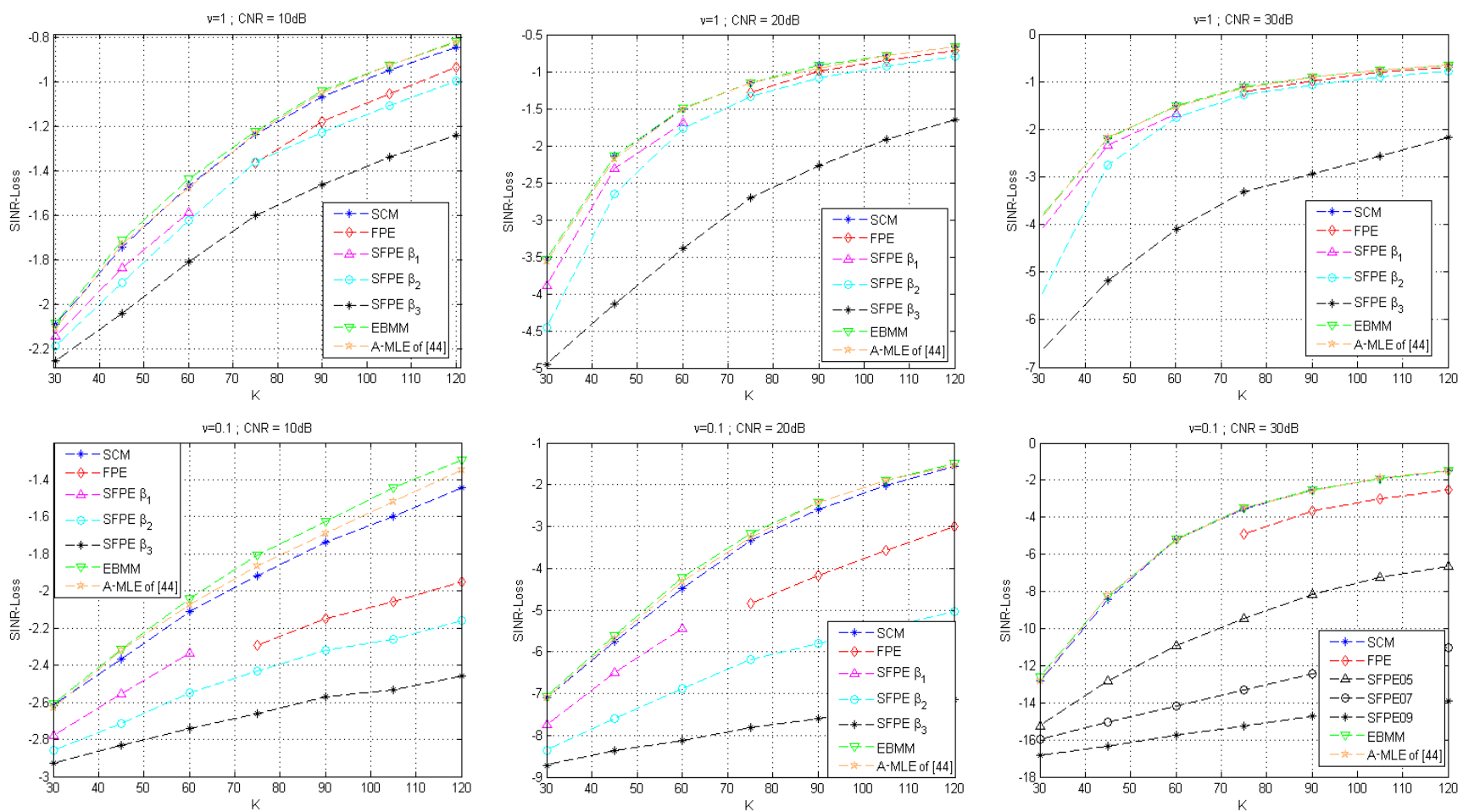

Figure 5. Mean SINR-Loss of the adaptive LR filters built from various clutter subspace projector estimators: $\hat{\boldsymbol{\Pi}}_{S C M}$ (blue), $\hat{\boldsymbol{\Pi}}_{F P E}$ (red), $\hat{\boldsymbol{\Pi}}_{S F P E}$ for $\beta_{1}$ (magenta), $\beta_{2}$ (cyan) and $\beta_{3}$ (black), $\hat{\boldsymbol{\Pi}}_{E B M M}$ computed with Algorithm 2: EBMM (green), $\hat{\boldsymbol{\Pi}}_{A-M L E}$ computed with algorithm "A-MLE" from [44] (orange). The SINR-Loss is presented for various clutter configurations. Columns from the left to the right: $\mathrm{CNR}=10 \mathrm{~dB}, \mathrm{CNR}=20 \mathrm{~dB}, \mathrm{CNR}=30 \mathrm{~dB}$. Up row: $\nu=1$ (mildly heterogeneous clutter), Down row: $\nu=0.1$ (highly heterogeneous clutter).

for $\alpha_{i}>0$ and $z_{i}>0$, and equality is achieved if the $z_{i}$ 's are equal. Let $z_{i}=\frac{1+c_{i} x}{1+c_{i} x^{t}}$, we have

$$
\begin{aligned}
& \frac{\sum_{i=1}^{n} \alpha_{i} \log \left(\frac{1+c_{i} x}{1+c_{i} x^{t}}\right)}{\sum_{i=1}^{n} \alpha_{i}} \\
= & \frac{\sum_{i=1}^{n} \alpha_{i} \log \left(1+c_{i} x\right)-\sum_{i=1}^{n} \alpha_{i} \log \left(1+c_{i} x^{t}\right)}{\sum_{i=1}^{n} \alpha_{i}} \\
\leq & \log \left(\frac{\sum_{i=1}^{n} \alpha_{i} \cdot \frac{1+c_{i} x}{1+c_{i} x^{t}}}{\sum_{i=1}^{n} \alpha_{i}}\right) .
\end{aligned}
$$

Rearranging the terms leads to the inequality (30), with equality achieved at $x=x^{t}$, which corresponds to $z_{i}=1$, $\forall i=1, \ldots, n$.

We can now turn to the proof of Proposition 1:

Proof: Ignoring the constant term, $L\left(\tau_{k} \mid \mathbf{W}\right)$ can be written as:

$$
\begin{aligned}
& L\left(\tau_{k} \mid \mathbf{W}\right) \\
= & \sum_{m=1}^{R} \log \left(\tau_{k} \lambda_{m}+1\right)+\sum_{m=1}^{R} s_{k m}\left(1-\frac{\tau_{k} \lambda_{m}}{\tau_{k} \lambda_{m}+1}\right) \\
= & \sum_{m=1}^{R} \log \left(\tau_{k} \lambda_{m}+1\right)-\sum_{m=1}^{R} s_{k m} \frac{\tau_{k} \lambda_{m}}{\tau_{k} \lambda_{m}+1}+\text { const. }
\end{aligned}
$$

First, applying Eq.(29) of Lemma 6 to the second term of (33) (with $x$ parameterized as $\frac{\tau_{k} \lambda_{m}}{\tau_{k} \lambda_{m}+1}$ ) yields

$$
\begin{aligned}
& L\left(\tau_{k} \mid \mathbf{W}^{t}\right) \\
\leq & \sum_{m=1}^{R} \log \left(\tau_{k} \lambda_{m}+1\right)+\text { const. } \\
& -\sum_{m=1}^{R} s_{k m} \frac{\tau_{k}^{t} \lambda_{m}}{\tau_{k}^{t} \lambda_{m}+1}\left(1+\log \tau_{k} \lambda_{m}-\log \left(\tau_{k} \lambda_{m}+1\right)\right) \\
\leq & \sum_{m=1}^{R}\left(s_{k m} \frac{\tau_{k}^{t} \lambda_{m}}{\tau_{k}^{t} \lambda_{m}+1}+1\right) \log \left(1+\lambda_{m} \tau_{k}\right) \\
& -\left(\sum_{m=1}^{R} s_{k m} \frac{\tau_{k}^{t} \lambda_{m}}{\tau_{k}^{t} \lambda_{m}+1}\right) \log \tau_{k}+\text { const. }
\end{aligned}
$$

where the equality is achieved at $\tau_{k}=\tau_{k}^{t}$. This upperbound is convex in $\tilde{\tau}_{k} \triangleq \log \tau_{k}$, but the minimizer cannot be computed in closed-form. Denote

$$
\alpha_{k m}=s_{k m} \frac{\tau_{k}^{t} \lambda_{m}}{\tau_{k}^{t} \lambda_{m}+1}+1 \text { and } \beta_{k}=\sum_{m=1}^{R} s_{k m} \frac{\tau_{k}^{t} \lambda_{m}}{\tau_{k}^{t} \lambda_{m}+1}
$$


upperbound in (34), which leads to:

$$
\begin{aligned}
& L\left(\tau_{k} \mid \mathbf{W}\right) \\
\leq & \sum_{m=1}^{R} \alpha_{k m} \log \left(1+\lambda_{m} \tau_{k}\right)-\beta_{k} \log \tau_{k}+\text { const. } \\
\leq & \left(\sum_{m=1}^{R} \alpha_{k m}\right) \log \left(\frac{\sum_{m=1}^{R} \alpha_{k m} \frac{1+\lambda_{m} \tau_{k}}{1+\lambda_{m} \tau_{k}^{t}}}{\sum_{m=1}^{R} \alpha_{k m}}\right)-\beta_{k} \log \tau_{k} \\
& + \text { const. } \\
= & \left(\sum_{m=1}^{R} \alpha_{k m}\right) \log \left(\frac{\left(\sum_{m=1}^{R} \frac{\alpha_{k m} \lambda_{m}}{1+\lambda_{m} \tau_{k}^{t}}\right)}{\sum_{m=1}^{R} \alpha_{k m}} \tau_{k}+\frac{\sum_{m=1}^{R} \frac{\alpha_{k m}}{1+\lambda_{m} \tau_{k}^{t}}}{\sum_{m=1}^{R} \alpha_{k m}}\right) \\
& -\beta_{k} \log \tau_{k}+\text { const. }
\end{aligned}
$$

Ignoring the constant term, we arrive at the surrogate function $L\left(\tau_{k} \mid \tau_{k}^{t}, \mathbf{W}\right)$ defined in Proposition 1. Note that the equality still holds at $\tau_{k}=\tau_{k}^{t}$.

\section{APPENDIX B}

\section{ProOF OF PROPOSITION 2}

Proof: The surrogate function, ignoring the constant term, has the form

$$
L\left(\tau_{k} \mid \tau_{k}^{t}, \mathbf{W}^{t}\right) \triangleq a \log \left(b \tau_{k}+c\right)-\beta_{k} \log \tau_{k} .
$$

The gradient of this surrogate function is

$$
\frac{\partial L\left(\tau_{k} \mid \tau_{k}^{t}, \mathbf{W}\right)}{\partial \tau_{k}}=\frac{a b}{b \tau_{k}+c}-\beta_{k} \frac{1}{\tau_{k}}
$$

and a zero of the gradient can be solved in closed-form as

$$
\tau_{k}^{t+1}=\frac{\beta_{k} c}{\left(a-\beta_{k}\right) b} .
$$

Since

$$
a=\sum_{m=1}^{R} \alpha_{k m}=\sum_{m=1}^{R}\left(s_{k m} \frac{\tau_{k}^{t} \lambda_{m}}{\tau_{k}^{t} \lambda_{m}+1}+1\right)
$$

and

$$
\beta_{k}=\sum_{m=1}^{R} s_{k m} \frac{\tau_{k}^{t} \lambda_{m}}{\tau_{k}^{t} \lambda_{m}+1},
$$

we have $a-\beta_{k}=R$. Therefore,

$$
\tau_{k}^{t+1}=\frac{1}{R} \cdot \frac{\left(\sum_{m=1}^{R} s_{k m} \frac{\tau_{k}^{t} \lambda_{m}}{\tau_{k}^{t} \lambda_{m}+1}\right) \cdot\left(\sum_{m=1}^{R} \frac{\alpha_{k m}}{1+\lambda_{m} \tau_{k}^{t}}\right)}{\sum_{m=1}^{R} \frac{\alpha_{k m} \lambda_{m}}{1+\lambda_{m} \tau_{k}^{t}}} \geq 0 .
$$

It remains to be shown that $\tau_{k}^{t+1}$ is the unique minimizer of $L\left(\tau_{k} \mid \tau_{k}^{t}, \mathbf{W}^{t}\right)$ on $\mathbb{R}_{+}$.

First assume that $\beta_{k} \neq 0$. Since $c$ is positive, $L\left(\tau_{k} \mid \tau_{k}^{t}, \mathbf{W}^{t}\right) \rightarrow+\infty$ as $\tau_{k} \rightarrow 0$. Besides, as $a>\beta_{k}$, $L\left(\tau_{k} \mid \tau_{k}^{t}, \mathbf{W}^{t}\right) \rightarrow+\infty$ as $\tau_{k} \rightarrow+\infty$. Since $L\left(\tau_{k}^{t+1} \mid \tau_{k}^{t}, \mathbf{W}^{t}\right)$ is finite, $\tau_{k}^{t+1}$ is the unique minimizer by the continuity of $L\left(\tau_{k} \mid \tau_{k}^{t}, \mathbf{W}^{t}\right)$. If $\beta_{k}=0$, then $\tau_{k}^{t+1}=0$. Function $a \log \left(b \tau_{k}+c\right)$ is monotonically increasing on $\mathbb{R}_{+}$, therefore $\tau_{k}^{t+1}=0$ is the unique minimizer. Moreover, as $L\left(\tau_{k} \mid \tau_{k}^{t}, \mathbf{W}^{t}\right)$ is a one-dimensional function, it has to be quasi-convex [50].

\section{APPENDIX C}

PROOF OF PROPOSITION 3

Proof: By the matrix inversion lemma we have the following equality:

$$
\begin{aligned}
\left(\tau_{k} \boldsymbol{\Sigma}+\mathbf{I}\right)^{-1} & =\mathbf{I}-\mathbf{W}\left(\tau_{k}^{-1} \mathbf{I}+\mathbf{W}^{H} \mathbf{W}\right)^{-1} \mathbf{W}^{H} \\
& =\mathbf{I}-\mathbf{W}\left(\tau_{k}^{-1} \mathbf{I}+\tilde{\boldsymbol{\Sigma}}\right)^{-1} \mathbf{W}^{H} .
\end{aligned}
$$

where $\tilde{\boldsymbol{\Sigma}} \triangleq \mathbf{W}^{H} \mathbf{W}$. Therefore, the second term of $L\left(\mathbf{W} \mid \tau_{k}\right)$ can be written as

$$
\begin{aligned}
& \mathbf{z}_{k}^{H}\left(\tau_{k} \boldsymbol{\Sigma}+\mathbf{I}\right)^{-1} \mathbf{z}_{k} \\
= & \mathbf{z}_{k}^{H} \mathbf{z}_{k}-\mathbf{z}_{k}^{H} \mathbf{W}\left(\tau_{k}^{-1} \mathbf{I}+\tilde{\boldsymbol{\Sigma}}\right)^{-1} \mathbf{W}^{H} \mathbf{z}_{k},
\end{aligned}
$$

which is jointly concave in $\{\mathbf{W}, \tilde{\boldsymbol{\Sigma}}\}$. The differential of Eq. (37) can be computed according to Eq. (38).

The concavity of (37) implies that it can be upperbounded by its first order Taylor expansion around the pair $\left\{\mathbf{W}^{t}, \tilde{\mathbf{\Sigma}}^{t}\right\}$, i.e.,

$$
\begin{aligned}
& \mathbf{z}_{k}^{H}\left(\tau_{k} \boldsymbol{\Sigma}+\mathbf{I}\right)^{-1} \mathbf{z}_{k} \\
\leq & -\operatorname{Tr}\left(\mathbf{z}_{k} \mathbf{z}_{k}^{H} \mathbf{W}^{t}\left(\tau_{k}^{-1} \mathbf{I}+\tilde{\boldsymbol{\Sigma}}^{t}\right)^{-1} \mathbf{W}^{H}\right) \\
& -\operatorname{Tr}\left(\left(\tau_{k}^{-1} \mathbf{I}+\tilde{\boldsymbol{\Sigma}}^{t}\right)^{-1}\left(\mathbf{W}^{t}\right)^{H} \mathbf{z}_{k} \mathbf{z}_{k}^{H} \mathbf{W}\right) \\
& +\operatorname{Tr}\left(\left(\tau_{k}^{-1} \mathbf{I}+\tilde{\boldsymbol{\Sigma}}^{t}\right)^{-1}\left(\mathbf{W}^{t}\right)^{H} \mathbf{z}_{k} \mathbf{z}_{k}^{H} \mathbf{W}^{t}\left(\tau_{k}^{-1} \mathbf{I}+\tilde{\boldsymbol{\Sigma}}^{t}\right)^{-1} \tilde{\boldsymbol{\Sigma}}\right) \\
& + \text { const. }
\end{aligned}
$$

Substituting $\tilde{\boldsymbol{\Sigma}}=\mathbf{W}^{H} \mathbf{W}$ into (39) leads to the following quadratic upperbound for $\mathbf{z}_{k}^{H}\left(\tau_{k} \boldsymbol{\Sigma}+\mathbf{I}\right)^{-1} \mathbf{z}_{k}$ :

$$
\operatorname{Tr}\left(\mathbf{W} \mathbf{H}_{k}^{t} \mathbf{W}^{H}\right)-\operatorname{Tr}\left(\mathbf{L}_{k}^{t} \mathbf{W}^{H}\right)-\operatorname{Tr}\left(\left(\mathbf{L}_{k}^{t}\right)^{H} \mathbf{W}\right),
$$

where

$$
\mathbf{H}_{k}^{t}=\left(\tau_{k}^{-1} \mathbf{I}+\tilde{\boldsymbol{\Sigma}}^{t}\right)^{-1}\left(\mathbf{W}^{t}\right)^{H} \mathbf{z}_{k} \mathbf{z}_{k}^{H} \mathbf{W}^{t}\left(\tau_{k}^{-1} \mathbf{I}+\tilde{\boldsymbol{\Sigma}}^{t}\right)^{-1}
$$

and

$$
\mathbf{L}_{k}^{t}=\mathbf{z}_{k} \mathbf{z}_{k}^{H} \mathbf{W}^{t}\left(\tau_{k}^{-1} \mathbf{I}+\tilde{\mathbf{\Sigma}}^{t}\right)^{-1} .
$$

As for the $\log \operatorname{det}(\cdot)$ term in $L\left(\mathbf{W} \mid \tau_{k}\right)$, we can apply the Sylvester's determinant theorem

$$
\log \operatorname{det}\left(\tau_{k} \mathbf{W} \mathbf{W}^{H}+\mathbf{I}\right)=\log \operatorname{det}\left(\tau_{k} \mathbf{W}^{H} \mathbf{W}+\mathbf{I}\right)
$$

and get the upperbound

$$
\operatorname{Tr}\left(\tau_{k}\left(\tau_{k} \tilde{\boldsymbol{\Sigma}}^{t}+\mathbf{I}\right)^{-1} \mathbf{W}^{H} \mathbf{W}\right)+\text { const. }
$$

by linearization over $\tilde{\boldsymbol{\Sigma}}$.

Together with (40) we have an upperbound (ignoring the constant terms) for $L\left(\mathbf{W} \mid \tau_{k}\right)$ at $\mathbf{W}^{t}$ as

$$
\begin{aligned}
& \operatorname{Tr}\left(\mathbf{W}\left(\sum_{k=1}^{K}\left(\tilde{\boldsymbol{\Sigma}}^{t}+\tau_{k}^{-1} \mathbf{I}\right)^{-1}\right) \mathbf{W}^{H}\right) \\
& +\sum_{k=1}^{K}\left(\operatorname{Tr}\left(\mathbf{W} \mathbf{H}_{k}^{t} \mathbf{W}^{H}\right)-\operatorname{Tr}\left(\mathbf{L}_{k}^{t} \mathbf{W}^{H}\right)-\operatorname{Tr}\left(\left(\mathbf{L}_{k}^{t}\right)^{H} \mathbf{W}\right)\right) \\
= & \operatorname{Tr}\left(\mathbf{W} \mathbf{H} \mathbf{W}^{H}\right)-\operatorname{Tr}\left(\mathbf{L} \mathbf{W}^{H}\right)-\operatorname{Tr}\left(\mathbf{L}^{H} \mathbf{W}\right),
\end{aligned}
$$




$$
\begin{aligned}
& \mathrm{d}\left(\mathbf{z}_{k}^{H}\left(\tau_{k} \boldsymbol{\Sigma}+\mathbf{I}\right)^{-1} \mathbf{z}_{k}\right)=-\mathrm{d}\left(\mathbf{z}_{k}^{H} \mathbf{W}\left(\tau_{k}^{-1} \mathbf{I}+\tilde{\mathbf{\Sigma}}\right)^{-1} \mathbf{W}^{H} \mathbf{z}_{k}\right) \\
= & -\left(\mathbf{z}_{k}^{H} \mathrm{~d} \mathbf{W}\left(\tau_{k}^{-1} \mathbf{I}+\tilde{\boldsymbol{\Sigma}}\right)^{-1} \mathbf{W}^{H} \mathbf{z}_{k}+\mathbf{z}_{k}^{H} \mathbf{W} \mathrm{d}\left(\tau_{k}^{-1} \mathbf{I}+\tilde{\boldsymbol{\Sigma}}\right)^{-1} \mathbf{W}^{H} \mathbf{z}_{k}+\mathbf{z}_{k}^{H} \mathbf{W}\left(\tau_{k}^{-1} \mathbf{I}+\tilde{\boldsymbol{\Sigma}}\right)^{-1} \mathrm{~d} \mathbf{W}^{H} \mathbf{z}_{k}\right) \\
= & -\left(\operatorname{Tr}\left(\left(\tau_{k}^{-1} \mathbf{I}+\tilde{\boldsymbol{\Sigma}}\right)^{-1} \mathbf{W}^{H} \mathbf{z}_{k} \mathbf{z}_{k}^{H} \mathrm{~d} \mathbf{W}\right)+\operatorname{Tr}\left(\mathbf{z}_{k} \mathbf{z}_{k}^{H} \mathbf{W}\left(\tau_{k}^{-1} \mathbf{I}+\tilde{\mathbf{\Sigma}}\right)^{-1} \mathrm{~d} \mathbf{W}^{H}\right)\right. \\
& \left.-\mathbf{z}_{k}^{H} \mathbf{W}\left(\tau_{k}^{-1} \mathbf{I}+\tilde{\boldsymbol{\Sigma}}\right)^{-1} \mathrm{~d}\left(\tau_{k}^{-1} \mathbf{I}+\tilde{\boldsymbol{\Sigma}}\right)\left(\tau_{k}^{-1} \mathbf{I}+\tilde{\mathbf{\Sigma}}\right)^{-1} \mathbf{W}^{H} \mathbf{z}_{k}\right) \\
= & -\left(\operatorname{Tr}\left(\left(\tau_{k}^{-1} \mathbf{I}+\tilde{\boldsymbol{\Sigma}}\right)^{-1} \mathbf{W}^{H} \mathbf{z}_{k} \mathbf{z}_{k}^{H} \mathrm{~d} \mathbf{W}\right)+\operatorname{Tr}\left(\mathbf{z}_{k} \mathbf{z}_{k}^{H} \mathbf{W}\left(\tau_{k}^{-1} \mathbf{I}+\tilde{\mathbf{\Sigma}}\right)^{-1} \mathrm{~d} \mathbf{W}^{H}\right)\right. \\
& \left.-\operatorname{Tr}\left(\left(\tau_{k}^{-1} \mathbf{I}+\tilde{\mathbf{\Sigma}}\right)^{-1} \mathbf{W}^{H} \mathbf{z}_{k} \mathbf{z}_{k}^{H} \mathbf{W}\left(\tau_{k}^{-1} \mathbf{I}+\tilde{\mathbf{\Sigma}}\right)^{-1} \mathrm{~d} \tilde{\mathbf{\Sigma}}\right)\right)
\end{aligned}
$$

where

$$
\mathbf{H}=\sum_{k=1}^{K}\left(\left(\tilde{\boldsymbol{\Sigma}}^{t}+\tau_{k}^{-1} \mathbf{I}\right)^{-1}+\mathbf{H}_{k}^{t}\right)
$$

and

$$
\mathbf{L}=\sum_{k=1}^{K} \mathbf{L}_{k}^{t}
$$

\section{REFERENCES}

[1] R. Schmidt, "Multiple emitter location and signal parameter estimation," IEEE Transactions on Acoustics, Speech and Signal Processing, vol. 34, no. 3, pp. 276-280, March 1986

[2] R. Roy and T. Kailath, "ESPRIT-Estimation of signal parameters via rotational invariant techniques," IEEE Transactions on Acoustics, Speech and Signal Processing, vol. 37, no. 7, pp. 984-995, July 1989.

[3] F. Robey, D. Fuhrmann, E. Kelly, and R. Nitzberg, "A CFAR adaptive matched filter detector," IEEE Transactions on Aerospace and Electronic Systems, vol. 28, no. 2, pp. 208 - 216, 1992.

[4] S. Kraut, L. Scharf, and L. McWhorter, "Adaptive subspace detectors," IEEE Transactions on Signal Processing, vol. 49, no. 1, pp. 1-16, january 2001.

[5] S. Haykin, Adaptive filter theory, ser. Prentice-Hall information and system sciences series. Prentice Hall, 2002.

[6] I. Kirsteins and D. Tufts, "Adaptive detection using a low rank approximation to a data matrix," IEEE Transactions on Aerospace and Electronic Systems, vol. 30, pp. 55 - 67, 1994.

[7] M. Rangaswamy, F. Lin, and K. Gerlach, "Robust adaptive signal processing methods for heterogeneous radar clutter scenarios," Signal Processing, vol. 84, pp. 1653 - 1665, 2004.

[8] P. Stoica and Y. Selen, "Model-order selection: a review of information criterion rules," IEEE Signal Processing Magazine, vol. 21, no. 4, pp. 36-47, July 2004.

[9] P. Perry and P. Wolfe, "Minimax rank estimation for subspace tracking," IEEE Journal of Selected Topics in Signal Processing, vol. 4, no. 3, pp. 504-513, June 2010.

[10] M. Gavish and D. Donoho, "The optimal hard threshold for singular values is," IEEE Transactions on Information Theory, vol. 60, no. 8, pp. 5040-5053, 2014

[11] J. Ward, "Space-time adaptive processing for airborne radar," Lincoln Lab., MIT, Lexington, Mass., USA, Tech. Rep., December 1994.

[12] L. E. Brennan and F. Staudaher, "Subclutter visibility demonstration," RL-TR-92-21, Adaptive Sensors Incorporated, Tech. Rep., March 1992.

[13] I. Reed, J. Mallett, and L. Brennan, "Rapid convergence rate in adaptive arrays," IEEE Transactions on Aerospace and Electronic Systems, vol. AES-10, no. 6, pp. 853 - 863, November 1974.

[14] A. Haimovich, "Asymptotic distribution of the conditional signal-tonoise ratio in an eigenanalysis-based adaptive array," IEEE Transactions on Aerospace and Electronic Systems, vol. 33, pp. 988 - 997, 1997.

[15] E. Ollila, D. Tyler, V. Koivunen, and H. Poor, "Complex elliptically symmetric distributions: Survey, new results and applications," IEEE Transactions on Signal Processing, vol. 60, no. 11, pp. 5597-5625, 2012.
[16] J. Billingsley, "Ground clutter measurements for surface-sited radar," MIT, Tech. Rep. 780, February 1993.

[17] E. Conte, A. De Maio, and C. Galdi, "Statistical analysis of real clutter at different range resolutions," IEEE Transactions on Aerospace and Electronic Systems, vol. 40, no. 3, pp. 903-918, July 2004

[18] M. Greco, F. Gini, and M. Rangaswamy, "Statistical analysis of measured polarimetric clutter data at different range resolutions," Radar, Sonar and Navigation, IEE Proceedings -, vol. 153, no. 6, pp. 473-481, December 2006

[19] E. Ollila, D. Tyler, V. Koivunen, and H. Poor, "Compound-Gaussian clutter modeling with an inverse gaussian texture distribution," IEEE Signal Processing Letters, vol. 19, no. 12, pp. 876-879, Dec 2012.

[20] M. Rangaswamy, I. Kirsteins, B. Freburger, and D. Tufts, "Signal detection in strong low rank Compound-Gaussian interference," in Proceedings of the 2000 IEEE Sensor Array and Multichannel Signal Processing Workshop. 2000., 2000, pp. 144-148.

[21] R. Raghavan, "Statistical interpretation of a data adaptive clutter subspace estimation algorithm," IEEE Transactions on Aerospace and Electronic Systems, vol. 48, no. 2, pp. 1370 - 1384, 2012.

[22] G. Ginolhac, P. Forster, F. Pascal, and J.-P. Ovarlez, "Performance of two low-rank STAP filters in a heterogeneous noise," IEEE Transactions on Signal Processing, vol. 61, no. 1, pp. 57-61, 2013.

[23] R. A. Maronna, "Robust $M$-estimators of multivariate location and scatter," Annals of Statistics, vol. 4, no. 1, pp. 51-67, January 1976.

[24] D. Tyler, "A distribution-free M-estimator of multivariate scatter," The Annals of Statistics, vol. 15, no. 1, pp. 234-251, 1987.

[25] F. Pascal, Y. Chitour, J. Ovarlez, P. Forster, and P. Larzabal, "Existence and characterization of the covariance matrix maximum likelihood estimate in Spherically Invariant Random Processes," IEEE Transactions on Signal Processing, vol. 56, no. 1, pp. 34 - 48, January 2008.

[26] E. Conte, A. D. Maio, and G. Ricci, "Recursive estimation of the covariance matrix of a compound-Gaussian process and its application to adaptive CFAR detection," IEEE Transactions on Signal Processing, vol. 50, no. 8, pp. 1908 - 1915, August 2002.

[27] F. Gini and A. Farina, "Vector subspace dete $\mathrm{n}$ in compound-Gaussian clutter. Part I: survey and new results," IEEE Transactions on Aerospace and Electronic Systems, vol. 38, no. 4, pp. 1295-1311, 2002.

[28] F. Gini, A. Farina, and M. Montanari, "Vector subspace detection in compound-Gaussian clutter. Part II: performance analysis," IEEE Transactions on Aerospace and Electronic Systems, vol. 38, no. 4, pp. 1312-1323, 2002

[29] F. Gini and M. Greco, "Covariance matrix estimation for CFAR detection in correlated heavy tailed clutter," Signal Processing, special section on SP with Heavy Tailed Distributions, vol. 82, no. 12, pp. 1847-1859, December 2002.

[30] E. Ollila and D. Tyler, "Distribution-free detection under complex elliptically symmetric clutter distribution," in 2012 IEEE 7th Sensor Array and Multichannel Signal Processing Workshop (SAM), June 2012, pp. 413-416.

[31] O. Besson and Y. Abramovich, "Adaptive detection in elliptically distributed noise and under-sampled scenario," IEEE Signal Processing Letters, vol. 21, no. 12, pp. 1531-1535, Dec 2014.

[32] M. Greco, S. Fortunati, and F. Gini, "Maximum likelihood covariance matrix estimation for complex elliptically symmetric distributions under 
mismatched condition," Signal Processing Elsevier, vol. 104, pp. 381386, Nov 2014.

[33] Y. Chen, A. Wiesel, and A. O. Hero, "Robust shrinkage estimation of high-dimensional covariance matrices," IEEE Transactions on Signal Processing, vol. 59, no. 9, pp. 4097-4107, 2011.

[34] F. Pascal, Y. Chitour, and Y. Quek, "Generalized robust shrinkage estimator and its application to stap detection problem," IEEE Transactions on Signal Processing, vol. 62, no. 21, pp. 5640-5651, Nov 2014.

[35] Y. Sun, P. Babu, and D. Palomar, "Regularized tyler's scatter estimator: Existence, uniqueness, and algorithms," IEEE Transactions on Signal Processing, vol. 62, no. 19, pp. 5143-5156, Oct 2014.

[36] E. Ollila and D. Tyler, "Regularized $M$-estimators of scatter matrix," IEEE Transactions on Signal Processing, vol. 62, no. 22, pp. 6059-6070, Nov 2014.

[37] A. Wiesel, "Unified framework to regularized covariance estimation in scaled Gaussian models," IEEE Transactions on Signal Processing, vol. 60, no. 1, pp. 29-38, 2012.

[38] — "Geodesic convexity and covariance estimation," IEEE Transactions on Signal Processing, vol. 60, no. 12, pp. 6182-6189, Dec 2012.

[39] I. Soloveychik and A. Wiesel, "Group symmetric robust covariance estimation," arXiv preprint arXiv:1412.2345, 2014.

[40] — - "Tyler's covariance matrix estimator in elliptical models with convex structure," IEEE Transactions on Signal Processing, vol. 62, no. 20, pp. 5251-5259, Oct 2014.

[41] Y. Sun, P. Babu, and D. Palomar, "Robust estimation of structured covariance matrix for heavy-tailed elliptical distributions," IEEE Transactions on Signal Processing, submited.

[42] A. Breloy, L. L. Magoarou, G. Ginolhac, F. Pascal, and P. Forster, "Maximum likelihood estimation of clutter subspace in non homogeneous noise context," in Proceedings of EUSIPCO, Marrakech, Morocco, September 2013.

[43] A. Breloy, G. Ginolhac, F. Pascal, and P. Forster, "Clutter subspace estimation in low rank heterogeneous noise context," IEEE Transactions on Signal Processing, vol. 63, no. 9, pp. 2173-2182, May 2015.

[44] _ - "Robust covariance matrix estimation in heterogeneous low-rank context," IEEE Transactions on Signal Processing, submitted.

[45] J. Manton, "Optimization algorithms exploiting unitary constraints," IEEE Transactions on Signal Processing, vol. 50, no. 3, pp. 635-650, Mar 2002.

[46] M. Razaviyayn, M. Hong, and Z. Luo, "A unified convergence analysis of block successive minimization methods for nonsmooth optimization," SIAM Journal on Optimization, vol. 23, no. 2, pp. 1126-1153, 2013.

[47] M. Steiner and K. Gerlach, "Fast converging adaptive processor or a structured covariance matrix," IEEE Transactions on Aerospace and Electronic Systems, vol. 36, no. 4, pp. 1115-1126, 2000.

[48] A. Aubry, A. De Maio, and V. Carotenuto, "Optimality claims for the fml covariance estimator with respect to two matrix norms," IEEE Transactions on Aerospace and Electronic Systems, vol. 49, no. 3, pp. 2055-2057, July 2013.

[49] K. Lange and H. Zhou, "MM algorithms for geometric and signomial programming," Mathematical programming, vol. 143, no. 1-2, pp. 339356, 2014.

[50] S. P. Boyd and L. Vandenberghe, Convex Optimization. Cambridge university press, 2004. 